\title{
Article \\ Fungal Chitosan-Derived Biomaterials Modified with Kalanchoe pinnata as Potential Hemostatic Agents-Development and Characterization
}

\author{
Julia Radwan-Pragłowska ${ }^{1, *}$, Łukasz Janus ${ }^{1}$, Marek Piątkowski ${ }^{1}{ }^{1}$, Aleksandra Sierakowska ${ }^{1}$, Tomasz Galek ${ }^{2}$, \\ Ernest Szajna ${ }^{3}$, Dariusz Bogdał ${ }^{1}$ and Mirosław Tupaj ${ }^{2}$ (D) \\ 1 Department of Biotechnology and Physical Chemistry, Faculty of Chemical Engineering and Technology, \\ Cracow University of Technology, Warszawska 24 Street, 31-155 Cracow, Poland; \\ lukasz.janus@doktorant.pk.edu.pl (Ł.J.); marek.piatkowski@pk.edu.pl (M.P.); \\ a.sierakowska3530@doktorant.pk.edu.pl (A.S.); pcbogdal@cyf-kr.edu.pl (D.B.) \\ 2 Faculty of Mechanics and Technology, Rzeszow University of Technology, Kwiatkowskiego 4 Street, \\ 37-450 Stalowa Wola, Poland; t.galek@prz.edu.pl (T.G.); mirek@prz.edu.pl (M.T.) \\ 3 Nano Prime, Metalowców 25, 39-200 Dębica, Poland; e.szajna@uce.com.pl \\ * Correspondence: j.radwan@doktorant.pk.edu.pl; Tel.: +48-12-628-27-76
}

\section{check for} updates

Citation: Radwan-Pragłowska, J.; Janus, Ł.; Piątkowski, M.;

Sierakowska, A.; Galek, T.; Szajna, E.;

Bogdał, D.; Tupaj, M. Fungal

Chitosan-Derived Biomaterials

Modified with Kalanchoe pinnata as Potential Hemostatic AgentsDevelopment and Characterization. Polymers 2021, 13, 1300. https:// doi.org/10.3390/polym13081300

Academic Editor: Luminita Marin

Received: 10 March 2021

Accepted: 12 April 2021

Published: 15 April 2021

Publisher's Note: MDPI stays neutral with regard to jurisdictional claims in published maps and institutional affiliations.

Copyright: (c) 2021 by the authors. Licensee MDPI, Basel, Switzerland. This article is an open access article distributed under the terms and conditions of the Creative Commons Attribution (CC BY) license (https:/ / creativecommons.org/licenses/by/ $4.0 /)$.

\begin{abstract}
Massive blood loss is still a great challenge for modern medicine. To stop the hemorrhage during the surgery or after injury apart from suturing or electrocoagulation, the most efficient method of hemostasis restoration is the use of hemostatic agents. Although there are numerous products on the market, there is still a need for biomaterials that are capable of fast and efficient bleeding management without affecting wound closure or embolism. Chitosan is known for its hemostatic activity; however, its quite poor mechanical properties and heterogenous chemical composition still needs some improvements to become superior compared to biological adhesives. The following study deals with the preparation and evaluation of chitosan-derived natural biomaterials containing Kalanchoe pinnata extract with the potential application as a blood-clotting agent. The materials were obtained under microwave-assisted conditions in two different forms (granules/dressing), whose chemical structure and morphology were studied. Their antioxidant properties have been proven. The chitosan-derived hemostatic agents exhibited superior blood sorption abilities and lack of cytotoxicity to L929 mouse fibroblasts. The study also showed the differences in biological properties depending on their preparation method. The potential mechanism of action was proposed as well as their potential in hemostasis revival.
\end{abstract}

Keywords: chitosan; hydrogel; biomaterials

\section{Introduction}

One of the biggest challenges for the modern medicine is massive blood loss, which is responsible for the highest number of deaths resulting from various injuries. Until now, no functional, Food and Drug Administration-approved artificial blood substitutes have been developed. Thus, the ability of fast and efficient inhibition of bleeding is an issue of a great importance. Currently, the most popular methods of hemostasis restoration include the use of polymeric sutures, electrocoagulation procedures, or application of hemostatic agents (HemA). The choice of HemA is especially understandable in the case of hemorrhages in the area of sensitive body parts such as nerves or medullary bone. Such materials are also willingly used in patients suffering from various hemostatic abnormalities. HemA minimizes the risk of peri-operative bleeding, decreases surgery duration, and provides support for the veins and arteries [1-3]. Importantly, hemostatic agents can be used not only by medics but also by individuals or military. HemA are commonly used in army in the battlefield when the bleedings come as a result of shooting or explosion. In such cases, 
HemA in the form of a powder or a plug is placed inside the deep wound and helps to stop the blood outflow from the veins before the patient's transport to the medical unit.

HemA can be divided into two main groups. The first one includes inorganic and non-resorbable materials, which are mostly zeolites. Due to their porous structure, they are capable of blood sorption; however, their use is associated with the risk of heat generation, leading to inflammatory reactions or even thermal injuries of the surrounding tissues, which is their main flaw. In addition, they must be removed from the wound site. Other examples include HemA based on bone wax. The second group comprises biodegradable materials, which are mostly prepared from various natural polymers such as microfibrillar collagen, gelatin, oxidized regenerated cellulose, polysaccharidic microspheres, and others $[1,4-8]$. These are mainly applied in the form of a dry matrix with a porous structure. Their mode of action is based on their capability of blood plasma sorption leading to the hyperconcentration of coagulation proteins as well as platelets. They undergo bioresorption process up to 10 weeks after application. However, their physicochemical and biological properties may affect the wound closure process, especially due to the significant volume increase as a result of swelling with blood. Alternatively, biological hemostatic agents such as thrombin, fibrin/ fibrinogen, or tranexamic acid are used. Bovine-origin thrombin acts as a natural catalyst for hemostasis restoration, since it helps to convert fibrinogen into fibrin. In turn, fibrin sealants can form a fibrin clot and induce hemostasis in only few minutes as a result of physiological cascade imitation. These biomaterials are used especially during cardiovascular surgeries associated with a high risk. Another group of HemA consists of adhesives that stop the bleeding only due to tissues sealing. Importantly, they do not absorb blood or affect coagulation cascade. Such biomaterials are based on polymers including poly(ethylene glycol), which turns into hydrogel after contact with blood or animal-derived albumin crosslinked with glutaraldehyde. Due to the fast mode of action (tissue sealing after 5-30 s), they are applied during surgeries dealing e.g., with aortic dissections. Sometimes, so-called dual HemA also can be found on the market. They provide support for natural clot formation and induce blood coagulation.

When choosing appropriate HemA, one must take under consideration the mode of action (biologically passive or active), form (powder, glue, patch), and possible side effects including allergic reactions coming from animal-derived substrates, embolism, hypotension, bloodborne disease, infection, immune-mediated coagulopathy, vascular thrombosis, anaphylaxis, and others. Since all of the aforementioned biomaterials used for hemostasis restoration have some downfalls or limitations in the scope of their application, there is still a great need for novel hemostatic agents' development characterized with improved both physicochemical and biological properties [1,9].

One of the most promising raw materials for HemA preparation is chitosan (CS), which is a natural polymer obtained from chitin-the second most abundant polysaccharide after cellulose. This polycation can be produced via the deacetylation of chitin using various biowaste from the food industry, agriculture, and others [9-12]. Commercially, $\mathrm{CS}$ is mostly prepared as a result of treating chitin with $\mathrm{NaOH}$ solution, leading to the removal of $N$-acetyl groups from its units. This process carried out on an industrial scale is proceeded under harsh conditions and relies on the use of shrimps, crabs, lobsters or squids' exoskeletons as a substrate, which are washed, dried, and homogenized. The first step of CS commercial production involves chitin demineralization performed using hydrochloric acid followed by deproteinization with sodium hydroxide of low concentration. To remove the color, a bleaching process is carried out with hydrogen peroxide. Finally, pre-treated chitin undergoes the deacetylation via treating with $40-50 \% \mathrm{NaOH}$ solution. The process is finished when the polymer contains at least $60 \%$ of glucosamine units in its structure. Alternatively, CS can be prepared via the biological route using bacteria-produced organic acids and specific enzymes such as proteases or chitin deacetylases. The enzymatic method is considered as environmentally friendly and enables preparation of the product with more uniform physicochemical properties such as average molecular mass, viscosity, and deacetylation degree. CS exhibits a significant resemblance to the naturally occurring 
extracellular matrix (ECM) components. This can be assigned to its chemical structure. Moreover, it is proven to be non-toxic and is well known for its biocompatibility. It undergoes biodegradation and exhibits antioxidant properties. It contains free hydroxyl and amino groups; thus, it may undergo various modifications, especially crosslinking. Moreover, it can be used for various enzymes immobilization such as laccase due to the possibility of amide bonds formation with carboxyl groups of the biomolecules. CS is also frequently used for the preparation of smart materials for various applications such as catalysis, remediation, agriculture, food packaging, and others. When designing a new, CS-based composite, polymer content must be chosen very carefully, since it affects many parameters of the final product such as active substance loading capacity [12]. It dissolves in aqueous solutions with $\mathrm{pH}$ below 6.3. Amino groups present in glucosamine (deacetylated units) are responsible for many attractive properties of CS such as antibacterial activity or chelating capability. CS may also interact with cell membrane components and has a hemostatic effect. It enhances cells adhesion and proliferation. CS may form physical and chemical hydrogels capable of aqueous solutions sorption. Native CS can be used as a powder, coated bandage, or patch to prevent bleeding. To increase its mechanical properties and enhance ability to hemostasis restoration, the polymer can be crosslinked to form three-dimensional, spacious structure. However, this leads to the loss of free amino groups, which may have a negative effect on its overall biological performance [9,13-17].

CS hemostatic agents' mode of action in the first way is based on its ability to absorb blood plasma and interact with erythrocytes, leading to their coagulation. The polymer swells with aqueous fraction of the blood, therefore concentrating its components, which take place in hemostasis reviewing. CS may enhance red blood cells adhesion and coagulation due to the absorption of proteins present in blood such as fibrinogen and helps in clot formation, yet still it does not explain its hemostatic activity [9]. This polysaccharide induces platelets adhesion followed by aggregation and finally activation, which is crucial during hemorrhage managing. It has been shown that CS may affect platelets spreading and adhesion stability, since it can generate various intracellular signal reactions. For example, it can activate glycoprotein IIb/IIIa or discharge thromboxane A2/ADP. In addition, some studies prove that platelets after direct contact with CS exhibit a higher expression of integrin $\alpha 2 \beta 3$. Finally, due to its ability of ions' chelation, CS may bind $\mathrm{Ca}^{2+}$ ions that are responsible for actin skeleton activation $[9,10]$. From this point of view, it must be acknowledged that to provide sufficient hemostatic activity of CS-based HemA, two main factors must be taken under consideration, namely sorption abilities, which are a result of the hydrophilic nature of the material due to certain functional groups such as hydroxyl, amino, or carboxylic as well as porous, open-cell, spatial architecture, which provides the possibility of water molecules migration inside the polymeric network, thus increasing swelling capability [4-9].

To enhance the biological activity of the biomaterials, various methods can be applied. These include blends formation with other polymers and biopolymers or inorganic substrates as well as doping with nanoparticles. Nevertheless, the addition of an extra component may deteriorate the biodegradation/bioresorption rate of the material or affect its mechanical durability. CS by itself exhibits some interesting features. One of them is antioxidant activity and positive impact on cells proliferation. Bioactivity is a highly desired property for modern HemA. Kalanchoe pinnata is a Madagascar-origin plant whose leaves and juice show strong anti-inflammatory, bactericidal, fungicidal, and virucidal activity. It perfectly regenerates all kinds of skin lesions. Moreover, it is known for its antioxidant and hepatoprotective properties and thus constitutes a great natural alternative to currently applied supporting drugs [18-26].

In this paper, a novel strategy for bioactive HemA preparation is presented using Aspergillus niger-derived CS as a main substrate. The biopolymer was chemically crosslinked with amino acids followed by modification with Kalanchoe pinnata leaves extracts. Ready biomaterials were investigated over their chemical structure with the use of the Fourier transform infrared spectroscopy (FTIR) method, morphology (e.g., using Scanning Electron 
Microscopy), mechanical durability, swelling properties, and antioxidant activity. Finally, the products were confirmed to be non-toxic to the L929 mouse fibroblasts cell line.

\section{Materials and Methods}

\subsection{Materials}

For the experiments, fungal chitosan from Aspergillus niger was used with the average molecular mass 890,000 , deacetylation degree $\geq 90 \%$, ash content $\leq 1.0 \%$, viscosity 100-300 cps, water content $\leq 8.0 \%$, As $\leq 1 \mathrm{mg} / \mathrm{kg}, \mathrm{Pb} \leq 0.5 \mathrm{mg} / \mathrm{kg}, \mathrm{Hg} \leq 0.1 \mathrm{mg} / \mathrm{kg}$, particle size $\leq 100$ mesh, E. coli microbial counts: absent, Salmonella spp.: absent, yeasts and molds: $\leq 100 \mathrm{CFU} / \mathrm{g}$ purified from glucan purchased from PolAura, Dywity, Poland. L-aspartic acid, L-glutamic acid, glycerin, and 1,2-propanediol were purchased from Po1Aura, Dywity, Poland. Kalanchoe pinnata extract was purchased from Ekamedica, Kozy, Poland. Simulated body fluid (SBF), Dulbecco's Modified Eagle's Medium, trypsin, fetal bovine serum, phosphate buffer solution, and penicillin/streptomycin solution (10\%) were purchased from PolGen. Multiwhole plates, T-75 flasks, sterile pipette tips, probes, and falcons were purchased from GenoPlast, Rokocin, Poland. XTT (2,3-Bis-(2-Methoxy-4-Nitro5-Sulfophenyl)-2H-Tetrazolium-5-Carboxanilide) assay (Roche), L929 mouse fibroblasts cell line, and lyophilized blood for scientific use only were purchased from Sigma-Aldrich, Poznań, Poland. Rhodamin, ethanol, mehtanol, and gallic acid were purchased from $\mathrm{POCH}$, Gliwice, Poland. Folin-Ciocalteu reagent was synthesized by standard procedure [27,28].

\subsection{Methods}

\subsubsection{Samples' Preparation}

To obtain chemically crosslinked chitosan-based hydrogels, fungal CS has been dissolved in 5\% solution of acetic acid, precipitated with 5\% sodium hydroxide solution, and lyophilized. To prepare the reaction mixture, $0.5 \mathrm{~g}$ of the precipitated CS was added in portions to the aqueous solution of $\mathrm{L}$-aspartic and L-glutamic acid $(\mathrm{pH}<6.3)$. Then, $30 \mathrm{~mL}$ of 1,2-propanediol were added to enable microwave radiation absorption. The glycol acted as a high boiling solvent. The reaction vessel ( $200 \mathrm{~mL}$ volume) was placed in the microwave reactor for $3 \mathrm{~min}$ with power set on $800 \mathrm{~W}$. After water evaporation, the crosslinking process has occurred, resulting in the CS color change. Then, the product was separated from the 1,2-propanediol and washed out from unreacted amino acids residues using distilled water until $\mathrm{pH}$ above 6 was achieved. To immobilize Kalanchoe pinnata extracts, each of the natural solutions was added to the swelling medium (distilled water). The samples (hydrogels) were immersed into the swelling solutions. The products were prepared in two different forms, namely a patch and beads. To prepare potential HemA in the form of a patch, the swollen hydrogels were placed in polypropylene boxes and lyophilized. To obtain hemostatic agents is the form of beads, the precipitated CS was dissolved in the $5 \%$ solution of amino acids and added dropwise to the $\mathrm{NaOH}$ solution using a syringe pump. Before lyophilization, cryoprotectant (glycol) was added to preserve the spherical structure. Samples description is given in the Table 1, while the components are presented in the Figure 1.

For the experiments, 4 different Kalanchoe pinnata extracts were used. Commercial extract was added to the samples without any pre-treatment in the amout of $1 \% w / w$. To prepare fresh extracts, fresh leaves were cut, frozen, and lyophilized using a freeze dryer (Alpha 1-4, Donserv, Warszawa, Poland). Then, $1 \mathrm{~g}$ of lyophilized leaves was placed in $50 \mathrm{~mL}$ of ethanol solutions ( $50 \%$ and $95 \%$ ). The extraction was carried out for $24 \mathrm{~h}$ under dark conditions to prevent photodegradation. Additionally, the plant extract prepared in $95 \%$ ethanol was subjected to ultrasounds using an ultrasonic bath $(1 \mathrm{~h})$. 
Table 1. Samples description and composition.

\begin{tabular}{cccc}
\hline Sample & Glu:Asp, g:g & Lyophilization & Form \\
\hline Gel1-0 & $0.90: 0.10$ & No & Patch \\
Gel2-0 & $0.75: 0.25$ & No & Patch \\
Gel3-0 & $0.25: 0.75$ & No & Patch \\
Gel4-0 & $0.10: 0.90$ & No & Patch \\
Gel-1 & $0.9: 0.10$ & Yes & Patch \\
Gel-2 & $0.75: 0.25$ & Yes & Patch \\
Gel-3 & $0.25: 0.75$ & Yes & Patch \\
Gel-4 & $0.10: 0.90$ & Yes & Patch \\
Gel-1-B & $0.9: 0.10$ & Yes & Beads \\
Gel-2-B & $0.75: 0.25$ & Yes & Beads \\
Gel-3-B & $0.25: 0.75$ & Yes & Beads \\
Gel-4-B & $0.10: 0.90$ & Yes & Beads \\
\hline
\end{tabular}
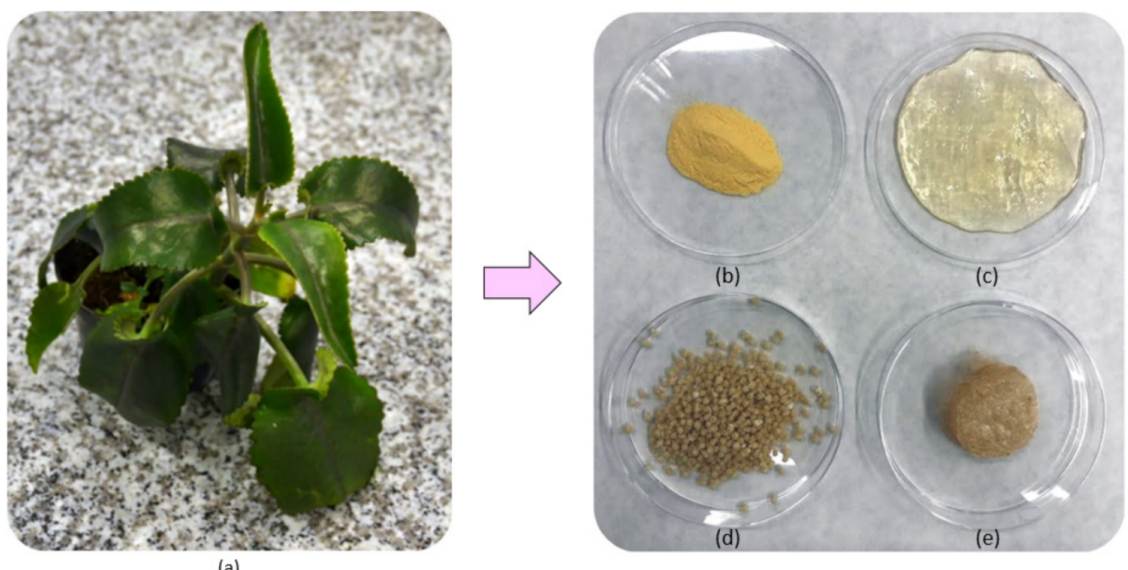

Figure 1. Photographs of (a) Kalanchoe pinnata plant used for extracts preparation (b) native chitosan (CS) (c) crosslinked hydrogel; (d) ready hemostatic agent in the form of beads; (e) hemostatic agent in the form of a patch.

\subsubsection{Fourier Transform Infrared Spectroscopy (FTIR) Study}

FTIR analysis was carried out using a Thermo Nicolet Nexus 470 FT-IR spectrometer from Thermo Fisher Scientific, Waltham, MA, USA equipped with an attenuated total reflection (ATR) diamond adapter. For the analysis, $50 \mathrm{mg}$ of each dried sample was used. The FTIR technique is used for determining the deacetylation degree. This method uses a characteristic absorption band corresponding to the amide group of the $N$-acetylglucosamine unit and a reference band that occurs in both glucosamine and $N$-acetylglucosamine units. These bands are visible at wavenumbers of $1655 \mathrm{~cm}^{-1}$ and $2870 \mathrm{~cm}^{-1}$. They correspond to the amounts of amide and hydroxyl groups in chitosan, respectively. The degree of deacetylation is calculated according to the equation:

$$
\mathrm{DD} \%=100-\frac{\mathrm{A}_{1655}}{\mathrm{~A}_{2870}} \times \frac{100}{1.33}
$$

where:

DD-deacetylation degree, $\%$.

$\mathrm{A}_{1655}$ - band absorption field at the wavenumber equal to $1635 \mathrm{~cm}^{-1}$.

$\mathrm{A}_{2870}$-band absorption field at the wavenumber equal to $3324 \mathrm{~cm}^{-1}$.

\subsubsection{Viscosity Study}

The viscosity of the samples and raw CS was determined using Brookfield RV rotational viscometer DV-II+ Pro. Viscosity of the native CS was measured by preparing 1\% 
polymer solution in acetic acid. Samples after crosslinking (fresh hydrogels) and samples after lyophilization (ready products) were swollen with distilled water. During the experiments, various spindles were used that enabled the evaluation of crosslinked samples.

\subsubsection{Morphology Study}

The samples were investigated by the Scanning Electron Microscopy (SEM) method. SEM images were prepared using a high-resolution MIRA Scanning Electron Miscroscope purchased from Tescan, Brno, Czech Republic. All samples were in a dried form. Samples' roughness was determined using Fiji image J software. The morphology of swollen samples was investigated using an inverted microscope (Delta Optical, Zielona Góra, Poland) and an epifluorescence adapter. For the analysis, materials were swollen with aqueous solution of rhodamine.

\subsubsection{Natural Extracts' Properties Study}

Plant extracts were verified over their optical properties by preparing methanol solutions $(100 \times$ dilution), which were placed in quartz cuvettes $(1 \mathrm{~cm}$ optical length), and UV-Vis spectra were collected in the range between 200 and $1000 \mathrm{~nm}$ using a UV-Vis Agilent 8453 diode-array spectrophotometer. Fluorescence spectra were collected in the range between 620 and $780 \mathrm{~nm}$ using a Jasco FP-750 spectrofluorimeter. The excitation wavelength was $365 \mathrm{~nm}$, whereas the optical slit for excitation and emission was $5 \mathrm{~nm}$. To determine phenolic and polyphenolic compounds content, the colorimetric method was used. For this purpose, Folin-Ciocalteu reagent was used. To proceed with the test, gallic acid as a standard was applied. The content of polyphenolic compounds was presented as $\mu \mathrm{g}$ per $1 \mathrm{~mL}$ of an extract. The test principle is based on the color change to dark blue. The measurements were performed at $750 \mathrm{~nm}$. As a medium, ammonium buffer solution $(\mathrm{pH}=10)$ was used $[27,28]$.

Antioxidant properties study were carried out by the DPPH (2,2-diphenyl-1-picrylhydrazyl) method. Test duration was $1 \mathrm{~h}$. The spectra were collected at $517 \mathrm{~nm}$ using UV-Vis spectrophotometer. For the experiments, freshly prepared DPPH solution in $95 \%$ ethanol with the concentration of $25 \mathrm{mg} / \mathrm{L}$ was used, which was stored under low temperature $\left(5^{\circ} \mathrm{C}\right)$ in the darkness.

\subsubsection{Swelling Properties Study}

Swelling properties studies were carried out using three different swelling media, namely distilled water, SBF, and blood liophylizate, which was subjected to a rehydratation process. During the tests, each lyophilized sample was weighed, placed in the medium for $5 \mathrm{~min}$, and weighed again. The swelling degree (SD) was calculated using following Equation (1):

$$
\mathrm{SD}=\mathrm{Ws}-\mathrm{Wd} / \mathrm{Wd} \cdot 100 \%
$$

where:

SD—swelling degree, $\%$.

Wd-weight of the dried sample, $g$.

Ws-weight of the swollen sample, g.

\subsubsection{Mechanical Properties Study}

Mechanical durability of the biomaterials was determined by tensile strength (TS) parameter measurement. All samples were prepared in the shape of a so-called "dog bone" according to PN-EN ISO 527:1998 standard for plastics mechanical properties study. For this purpose, swollen samples were freeze-dried in the molds printed using a 3D printer (Ender, Warsaw, Poland) using poly(lactic acid) filament. Dried and shaped samples were swollen with SBF and had a thickness of $4.0 \mathrm{~mm}$, overall length of $150 \mathrm{~mm}$, and measuring part width of $10 \mathrm{~mm}$. 


\subsubsection{Cytotoxicity Study}

Cytotoxicity was evaluated by quantitative and qualitative methods according to ISO 10993 standard for biomedical devices. For this purpose, L929 mouse fibroblasts were cultured under standard conditions (high humidity, $5 \% \mathrm{CO}_{2}, 37^{\circ} \mathrm{C}$ ). The cell culture medium was changed every $24 \mathrm{~h}$. Cells' proliferation assay based on the tetrazolium salt reduction (XTT assay) was carried out according to the producer's protocol (Roche) after $48 \mathrm{~h}$. The colorimetric reaction was investigated using a microplate reader at $450 \mathrm{~nm}$. To evaluate cytotoxcicity by direct method, the cells were seeded inside the 24-well plates containing $10 \mathrm{mg}$ of each sample. Their morphology was observed under an inverted microscope equipped with an epifluorescence adapter (Delta Optical, Zielona Góra, Poland) after $72 \mathrm{~h}$ of culture.

All experiments were performed in at least triplicate. Statistical analysis was performed by Excel software, and a $p<0.05$ value was found to be statistically significant.

\section{Results}

\subsection{Natural Extracts Investigation}

Photographs of the Kalanchoe pinnata extracts can be found in Figure 2. Figure 3 shows FTIR spectra of the Kalanchoe pinnata leaf, which is commercially available, as well as self-made plant extracts. Results are given in Table 2. The spectrum of the lyophilized and ground leaves shows bands typical for polysaccharides at $3324 \mathrm{~cm}^{-1}$ coming from hydroxyl groups and $2923 \mathrm{~cm}^{-1}$ and $2878 \mathrm{~cm}^{-1}$ coming from $-\mathrm{CH}_{2}$ and $-\mathrm{CH}_{3}$ moieties. There are also visible bands characteristic for $-\mathrm{CO}-$ stretching vibrations at $1006 \mathrm{~cm}^{-1}$. The presence of polyphenolic compounds is confirmed by the bands in the regions from $1722 \mathrm{~cm}^{-1}$ to $1400 \mathrm{~cm}^{-1}$ and around $1000 \mathrm{~cm}^{-1}$ typical for $\mathrm{C}=\mathrm{C}$ aromatic ring stretching and $-\mathrm{C}=\mathrm{O}$ stretching vibrations $[29,30]$. However, their intensity is low. The FTIR spectrum of the commercial extract shows bands coming from carboxyl groups at $3187 \mathrm{~cm}^{-1}$ of quite low intensity as well as bands at $2943 \mathrm{~cm}^{-1}$ and $2875 \mathrm{~cm}^{-1}$ that are typical for $-\mathrm{CH}_{2}-$ and $-\mathrm{CH}_{3}$. A band of high intensity coming from $\alpha, \beta$-unsaturated aliphatic ester groups is present at $1713 \mathrm{~cm}^{-1}$, which is typical for flavonoids. The band at $1591 \mathrm{~cm}^{-1}$ can be assigned to $C=C$ aromatic ring stretching vibrations. The band at $1394 \mathrm{~cm}^{-1}$ comes from $\mathrm{C}=\mathrm{C}$ stretching vibrations. Bands at $1183 \mathrm{~cm}^{-1}$ and $1089 \mathrm{~cm}^{-1}$ can be assigned to $\mathrm{C}-\mathrm{O}$ as well as C-C stretching vibrations (phenyl carbon). The spectrum of the extract prepared using $50 \%$ ethanol solution shows very similar bands; however, these are of different intensity. The broad band at $3351 \mathrm{~cm}^{-1}$ is typical for hydroxyl groups. Bands corresponding to $\mathrm{C}-\mathrm{H}$, $-\mathrm{CH}_{2-}$, and $-\mathrm{CH}_{3}$ at $3009 \mathrm{~cm}^{-1}, 2924 \mathrm{~cm}^{-1}$, and $2856 \mathrm{~cm}^{-1}$ suggest higher content of aliphatic chains in comparison to commercial extract. The region between $1720 \mathrm{~cm}^{-1}$ and $1000 \mathrm{~cm}^{-1}$ is typical for polyphenols. The aforementioned intense band comes from $\alpha, \beta-$ unsaturated aliphatic $-\mathrm{C}=\mathrm{O}$ groups. The band at $1599 \mathrm{~cm}^{-1}$ comes from $\mathrm{C}=\mathrm{C}$ stretching vibrations of aromatic rings. The band at $1396 \mathrm{~cm}^{-1}$ is typical for unsaturated double bonds. Finally, bands at $1176 \mathrm{~cm}^{-1}$ and $1092 \mathrm{~cm}^{-1}$ typical for C-O and C-C (phenyl carbon) are present. The spectrum of natural extract prepared by ethanol of higher concentration (4) shows a higher content of aliphatic chains (increased intensity of bands at $2923 \mathrm{~cm}^{-1}$ and $2858 \mathrm{~cm}^{-1}$ ). Importantly, the band coming from the $\mathrm{C}=\mathrm{C}$ aromatic ring and $\mathrm{C}=\mathrm{O}$ stretching vibrations shows a higher content of polyphenolic compounds suggesting the higher efficiency of its extraction process. The last spectrum presents extract prepared using $95 \%$ EtOH solution enhanced by ultrasounds. When comparing the spectra, it can be noticed that such an obtainment method results in the product characterized by the highest content of polyphenols which prove bands at $3308 \mathrm{~cm}^{-1}$ as well as at $1589 \mathrm{~cm}^{-1}[29,30]$. 


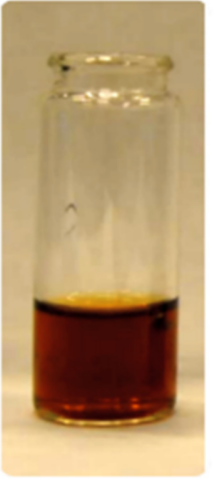

(a)

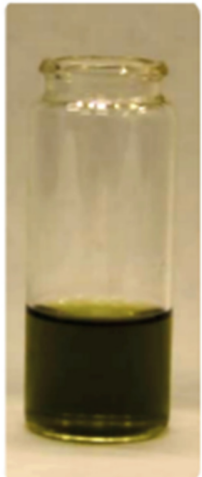

(b)

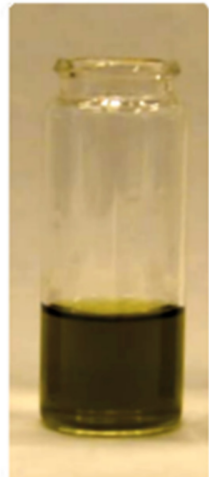

(c)

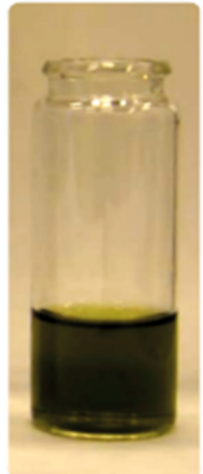

(d)

Figure 2. Photographs of the Kalanchoe pinnata extracts: (a) commercially available extract; (b) plant extract prepared using 50\% ethanol solution; (c) plant extract prepared using $95 \%$ ethanol solution; (d) plant extract prepared using 95\% ethanol solution and ultrasounds.

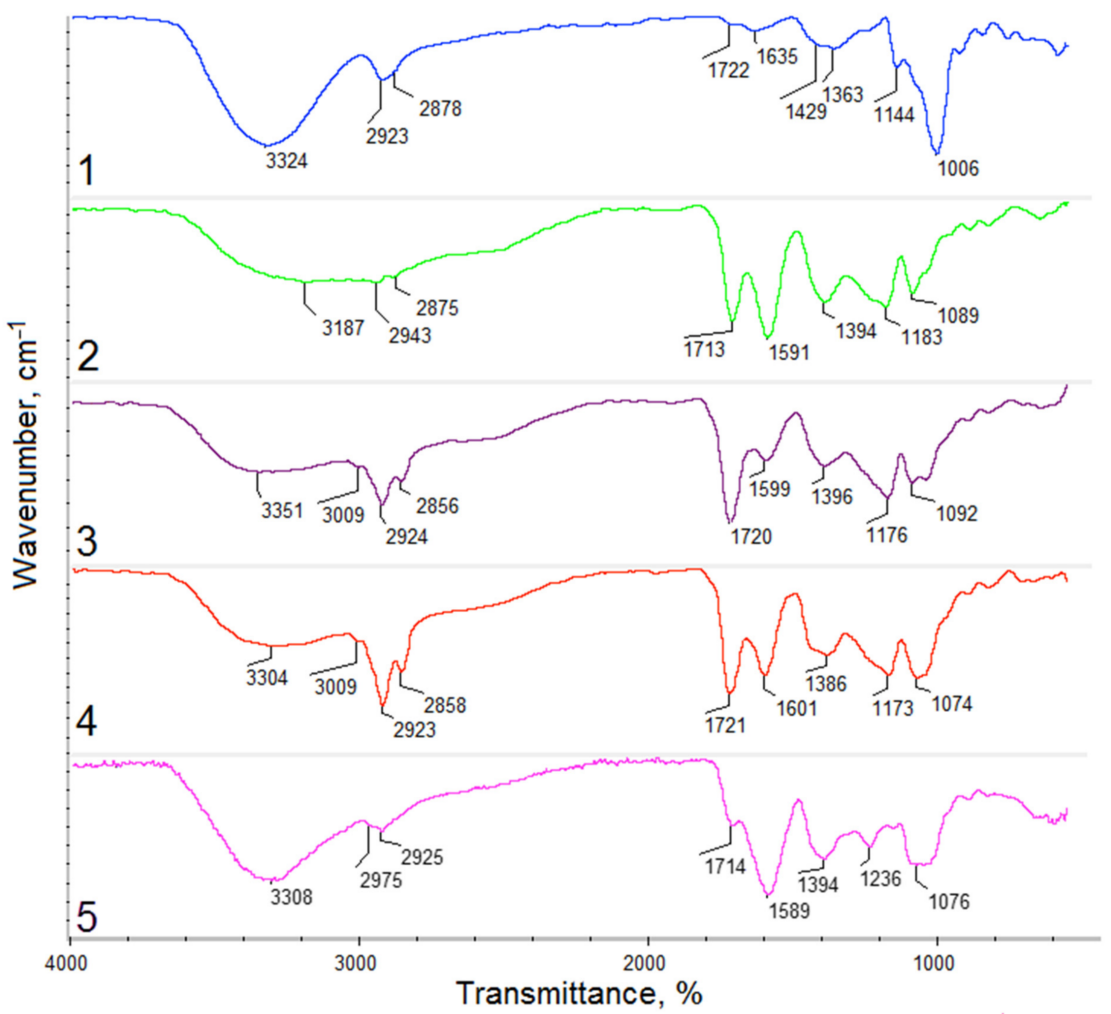

Figure 3. FTIR spectra of (1) Kalanchoe pinnata leaf; (2) commercially available extract; (3) plant extract prepared using 50\% ethanol solution; (4) plant extract prepared using 95\% ethanol solution; (5) plant extract prepared using $95 \%$ ethanol solution and ultrasounds.

To further investigate phenols, polyphenols, and other biologically relevant substances content, UV-Vis spectra were collected. Figure 4a shows UV-Vis spectrum of the commercial extract, which contains only one peak typical for auxochromes. The maximum peak intensity at $270 \mathrm{~nm}$ corresponds to $\mathrm{n} \mu \rightarrow \pi^{*}$ transitions. The spectra of the extracts prepared using ethanol solutions differ from the commercial one and exhibit more peaks that are associated with the presence of the aromatic compounds, especially polyphenols. Apart from the peaks with the maximum at $266 \mathrm{~nm}(50 \% \mathrm{EtOH}, 95 \% \mathrm{EtOH}$ and $95 \% \mathrm{EtOH} /$ ultrasounds), there are other signals typical for conjugated double bonds corresponding to $n \rightarrow \pi^{*}$ and $\pi \rightarrow \pi^{*}$ transitions [26]. 
Table 2. Fourier transform infrared spectroscopy (FTIR) analysis results.

\begin{tabular}{|c|c|c|c|c|c|c|}
\hline Sample & $\begin{array}{r}\mathrm{O}-\mathrm{H} \\
\mathrm{cm}^{-1}\end{array}$ & $\begin{array}{c}\mathrm{C}-\mathrm{H} \\
\mathrm{cm}^{-1}\end{array}$ & $\begin{array}{c}\mathrm{C}=\mathrm{C}-\mathrm{H} \\
\mathrm{cm}^{-1}\end{array}$ & $\begin{array}{l}\mathrm{C}=\mathrm{O} \\
\mathrm{cm}^{-1}\end{array}$ & $\begin{array}{l}\mathrm{C}=\mathrm{C} \\
\mathrm{cm}^{-1}\end{array}$ & $\begin{array}{r}\mathrm{C}-\mathrm{O} \\
\mathrm{cm}^{-1}\end{array}$ \\
\hline Kalanchoe pinnata leaf & 3324 & $\begin{array}{l}2923 \\
2878\end{array}$ & - & 1722 & 1429 & 1006 \\
\hline Commercially available extract & 3187 & $\begin{array}{l}2943 \\
2875\end{array}$ & - & 1713 & $\begin{array}{l}1591 \\
1394 \\
1089\end{array}$ & 1118 \\
\hline $\begin{array}{l}\text { Plant extract prepared using } 50 \% \\
\text { ethanol solution }\end{array}$ & 3351 & $\begin{array}{l}2924 \\
2856\end{array}$ & 3009 & 1720 & $\begin{array}{l}1599 \\
1396 \\
1092\end{array}$ & 1176 \\
\hline $\begin{array}{l}\text { Plant extract prepared using } 95 \% \\
\text { ethanol solution }\end{array}$ & 3304 & $\begin{array}{l}2923 \\
2858\end{array}$ & 3009 & 1721 & $\begin{array}{l}1601 \\
1386 \\
1074\end{array}$ & 1173 \\
\hline $\begin{array}{l}\text { Plant extract prepared using } 95 \% \\
\text { ethanol solution and ultrasounds }\end{array}$ & 3308 & $\begin{array}{l}2975 \\
2925\end{array}$ & - & 1714 & $\begin{array}{l}1589 \\
1394 \\
1076\end{array}$ & 1236 \\
\hline
\end{tabular}

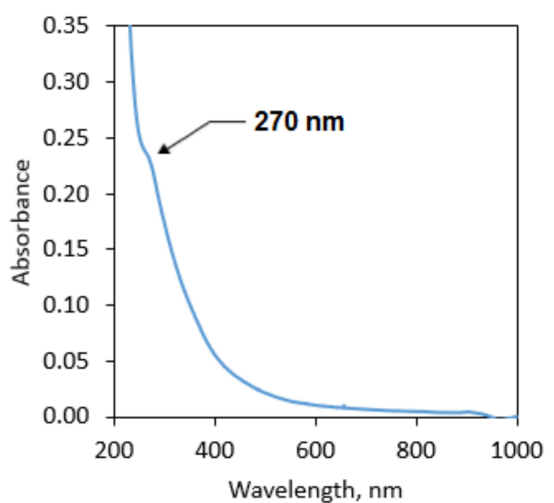

(a)

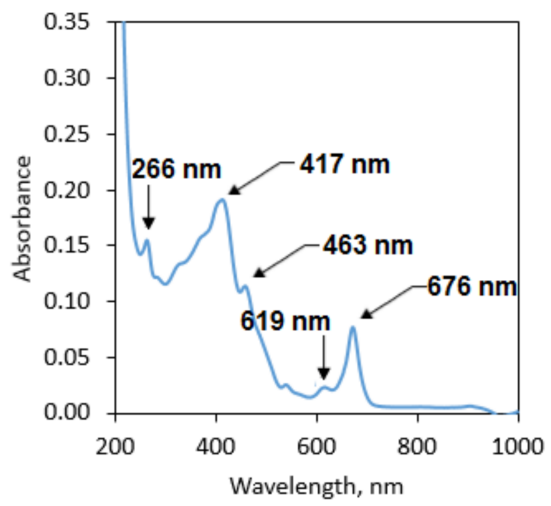

(c)

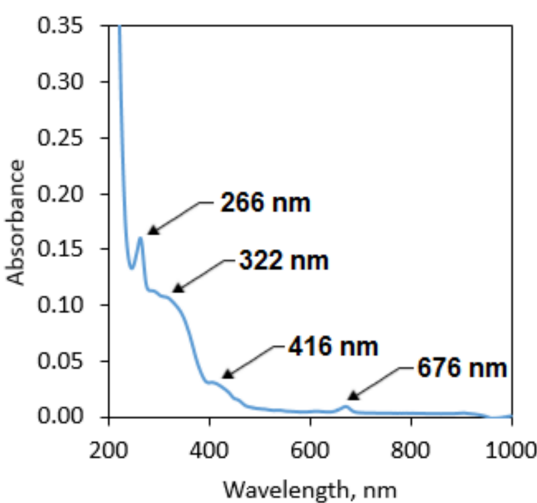

(b)

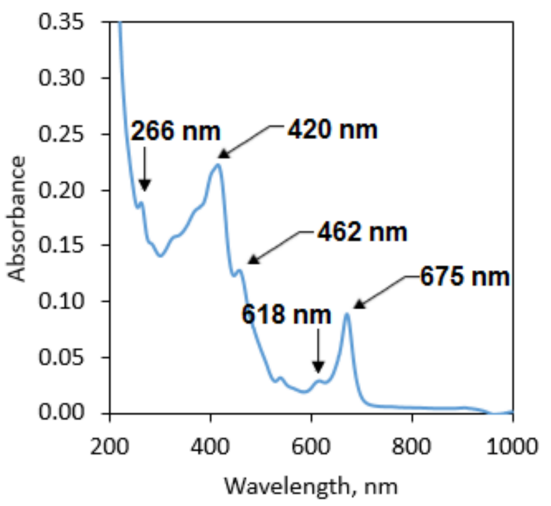

(d)

Figure 4. UV-Vis spectra of the natural extracts (a) commercially available extract; (b) plant extract prepared using 50\% EtOH solution; (c) plant extract prepared using 95\% EtOH solution; (d) plant extract prepared using 95\% EtOH solution and ultrasounds.

Figure 5 shows fluorescence spectra of the evaluated extracts. One may observe that all four samples exhibit similar peaks with the maximum at $669 \mathrm{~nm}$ (commercial extract), $668 \mathrm{~nm}$ (plant extract 50\% EtOH), $672 \mathrm{~nm}$ (plant extract 95\% EtOH), and $671 \mathrm{~nm}$ (plant extract $95 \% \mathrm{EtOH} /$ ultrasounds), respectively. The signals come from porphyrin dyes present in the Kalanchoe pinnata Chlorophyll $a$. Their intensity is similar, which suggests comparable content. 


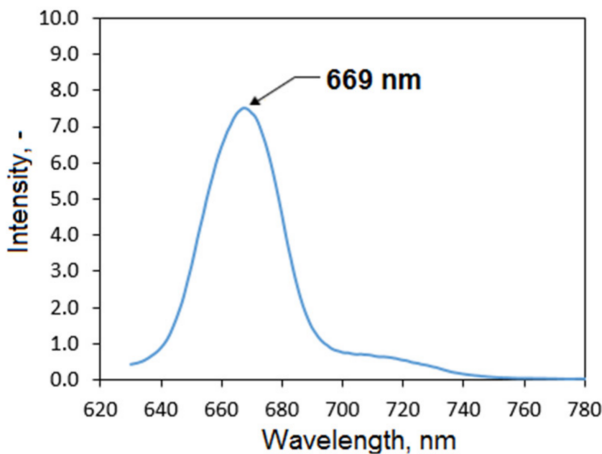

(a)

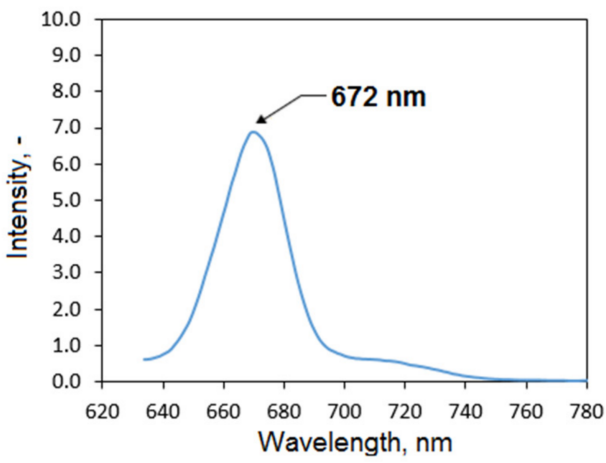

(c)

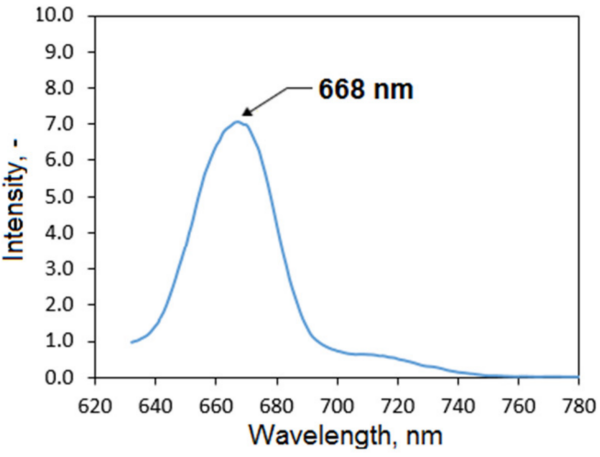

(b)

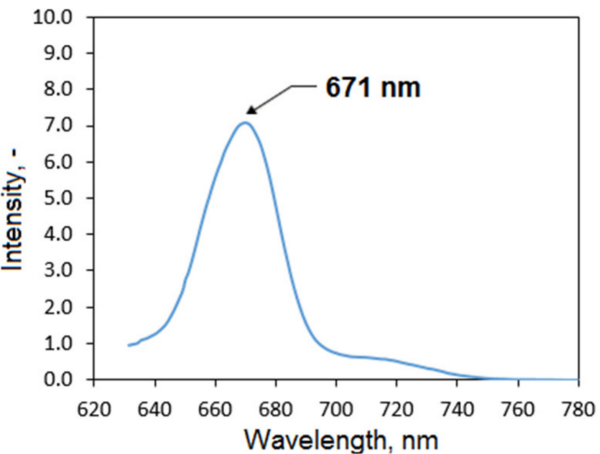

(d)

Figure 5. Fluorescence spectra of the natural extracts: (a) commercially available extract; (b) plant extract prepared using 50\% ethanol solution; (c) plant extract prepared using $95 \%$ ethanol solution; (d) plant extract prepared using 95\% ethanol solution and ultrasounds.

To investigate the total phenolic and polyphenolic compounds content in different extracts, the UV-Vis spectroscopic method using gallic acid as a standard was applied (Figure 6), which enables quantitative determination of the aforementioned substances $[27,28]$. The calibration plot prepared at $750 \mathrm{~nm}$ resulted in $\mathrm{R}^{2}=0.9995$. The study showed that the highest quantity of the organic substances responsible for antioxidant activity of the Kalanchoe pinnata can be found in the commercial extract (more than $80 \mu \mathrm{g} / 1 \mathrm{~mL}$ ) and can be considered as significantly different from other samples.

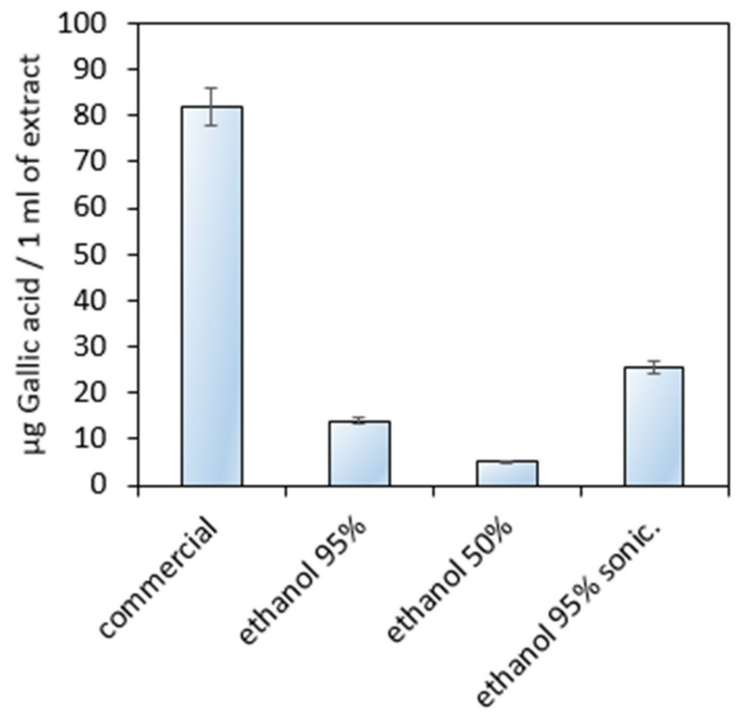

Figure 6. Phenols and polyphenols content investigation using Folin-Ciocalteu reagent. 


\subsection{Hemostatic Agents FTIR Analysis}

FTIR spectra of the raw fungal chitosan, Gel-1, Gel-2, Gel-3, and Gel-4 samples containing plant extract prepared using commercial Kalanchoe pinnata extract are given in Figure 7. FTIR results are presented in Table 3. The raw chitosan spectrum shown is typical for this polymer band. First, the band coming from free hydroxyl and amino groups with the maximum at $3359 \mathrm{~cm}^{-1}$ is visible. The presence of $\mathrm{NH}_{2}$ moieties is confirmed also by the bands at $1576 \mathrm{~cm}^{-1}$ and $1154 \mathrm{~cm}^{-1}$. The band at $1653 \mathrm{~cm}^{-1}$ corresponds to amide bonds present in acylated units of the CS. In addition, bands coming from glycosidic bonds as well as glucopyranose rings are visible at $1029 \mathrm{~cm}^{-1}$ and $885 \mathrm{~cm}^{-1}$ [12,15,16,31]. CS crosslinked with amino acids shows some significant changes in the bands' intensity. On the contrary to the commonly applied methods using glutaraldehyde as a crosslinking agent, there is no decrease in the free amino groups' intensity. Since amino groups are prone to react, the crosslinking process occurs between the carboxyl groups of L-aspartic and L-glutamic acid and amino groups present in glucosamine units, due to which bands coming from free amino groups are of higher intensity compared to the native CS $(1576,1755,1581,1573,1154$, 1148,1148 and $1154 \mathrm{~cm}^{-1}$, respectively). Importantly, when comparing collected spectra to other researchers' data, a significant difference in amino groups content is visible $[12,15,16]$. Covalent bonds formation proves bands typical for amides at 1658, 1633, 1645, and 1651 $\mathrm{cm}^{-1}$, respectively. In each case, wide overlapping bands coming from free hydroxyl and carboxyl groups at 3229, 3229, 3250, and $3208 \mathrm{~cm}^{-1}$ are visible [31]. The presence of Kalanchoe pinnata extract confirms bands at $1732 \mathrm{~cm}^{-1}$ and 1738 coming from carbonyl groups as well as bands around $1400 \mathrm{~cm}^{-1}$ coming from unsaturated carbon double bonds as well as bands around $1600 \mathrm{~cm}^{-1}$, which can be assigned to $\mathrm{C}=\mathrm{C}$ aromatic ring stretching vibrations [29,30]. All FTIR spectra exhibit also glycosidic bonds and glucopyranose rings typical for CS [31]. Basing on the obtained results, the proposed reaction scheme has been presented in Figure 8.

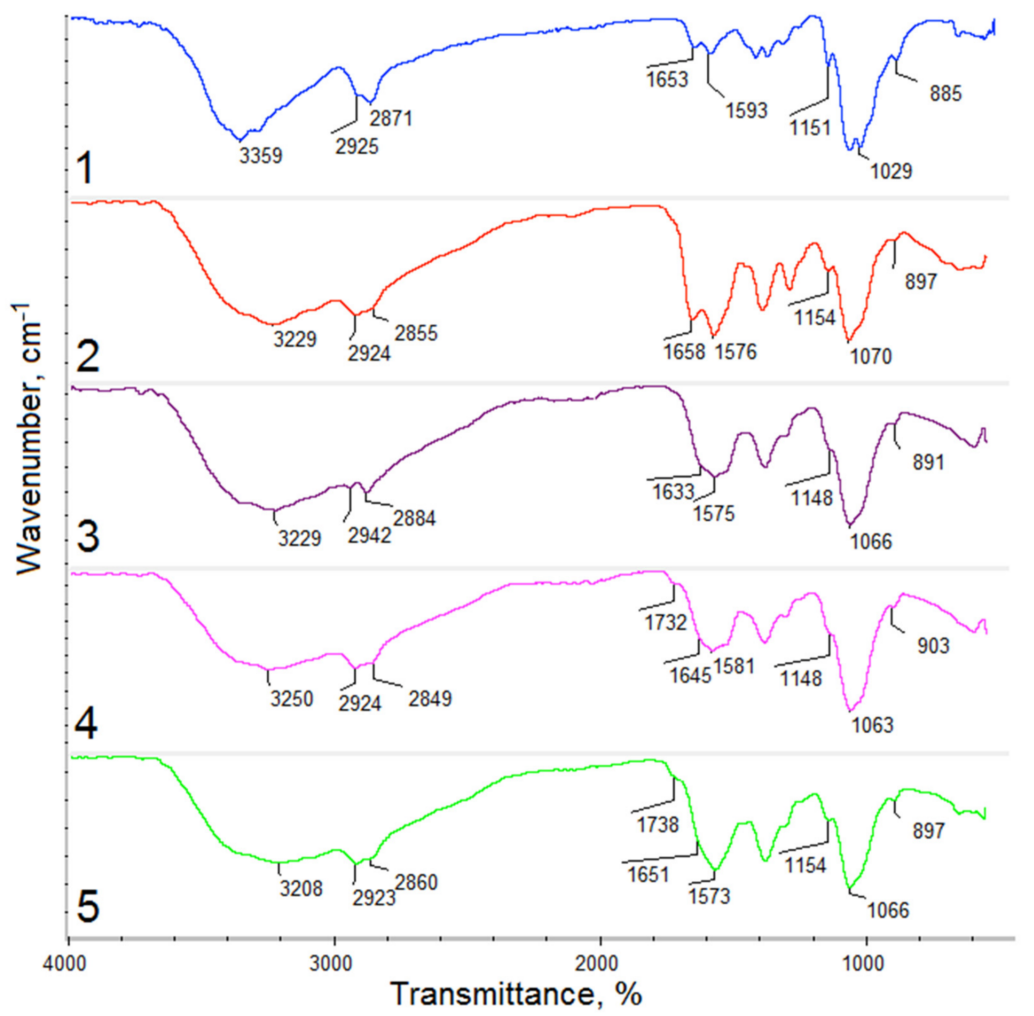

Figure 7. FTIR spectra of the raw fungal chitosan (1), Gel-1 (2), Gel-2 (3), Gel-3 (4), and Gel-4 (5) samples containing plant extract prepared using commercial Kalanchoe pinnata extract. 

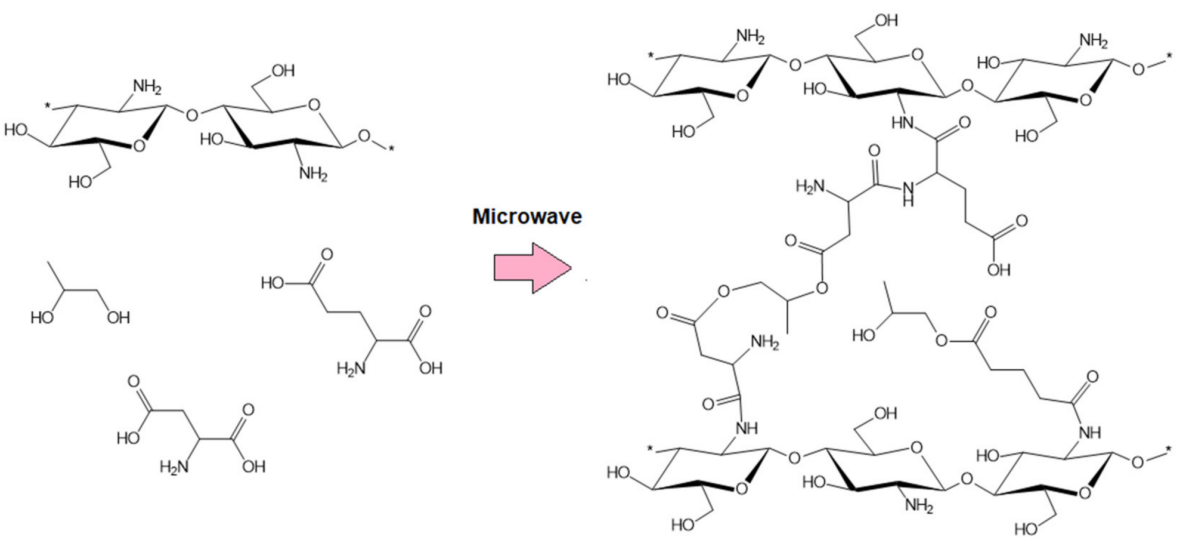

Figure 8. General scheme of hemostatic agent preparation $\left({ }^{*}\right.$-another polymeric unit)

Table 3. FTIR results.

\begin{tabular}{|c|c|c|c|c|c|c|}
\hline Sample & $\begin{array}{c}\mathrm{O}-\mathrm{H} \\
\mathrm{cm}^{-1}\end{array}$ & $\begin{array}{c}\mathrm{C}-\mathrm{H} \\
\mathrm{cm}^{-1}\end{array}$ & $\begin{array}{l}\mathrm{C}=\mathrm{O} \\
\mathrm{cm}^{-1}\end{array}$ & $\begin{array}{l}-\mathrm{NH}_{2} \\
\mathrm{~cm}^{-1}\end{array}$ & $\begin{array}{c}\mathrm{C}-\mathrm{O} \\
\mathrm{cm}^{-1}\end{array}$ & $\begin{array}{c}-\mathrm{COO}- \\
\mathrm{cm}^{-1}\end{array}$ \\
\hline \multirow{2}{*}{ Raw fungal chitosan } & \multirow{2}{*}{3359} & 2925 & \multirow{2}{*}{1653} & 1593 & 1029 & \multirow{2}{*}{ - } \\
\hline & & 2871 & & 1151 & 885 & \\
\hline \multirow{2}{*}{ Gel-1 } & \multirow{2}{*}{3229} & 2924 & \multirow{2}{*}{1658} & 1576 & 1070 & \multirow{2}{*}{ - } \\
\hline & & 2855 & & 1154 & 897 & \\
\hline \multirow{2}{*}{ Gel-2 } & \multirow{2}{*}{3229} & 2924 & \multirow{2}{*}{1658} & 1575 & 1066 & \multirow{2}{*}{ - } \\
\hline & & 2284 & & 1148 & 891 & \\
\hline \multirow{2}{*}{ Gel-3 } & \multirow{2}{*}{3250} & 2924 & \multirow{2}{*}{1645} & 1581 & 1063 & \multirow{2}{*}{1732} \\
\hline & & 2849 & & 1148 & 903 & \\
\hline \multirow{2}{*}{ Gel-4 } & \multirow{2}{*}{3208} & 2923 & \multirow{2}{*}{1651} & 1573 & 1066 & \multirow{2}{*}{1738} \\
\hline & & 2860 & & 1154 & 897 & \\
\hline
\end{tabular}

Raw CS is known for its favorable biological properties such as biocompatibility, biodegradability, mucoadhesiveness, antioxidant, and hemostatic activity. Importantly, most of them are related to the presence of free amino groups, which interact with negatively charged cell membranes, as given in Figure 9. Such chemical composition should provide sufficient blood components interaction and provide superior hemostatic activity comparing to other polymer-based biomaterials prepared using cellulose, poly(ethylene glycol), gelatin, and others [9].

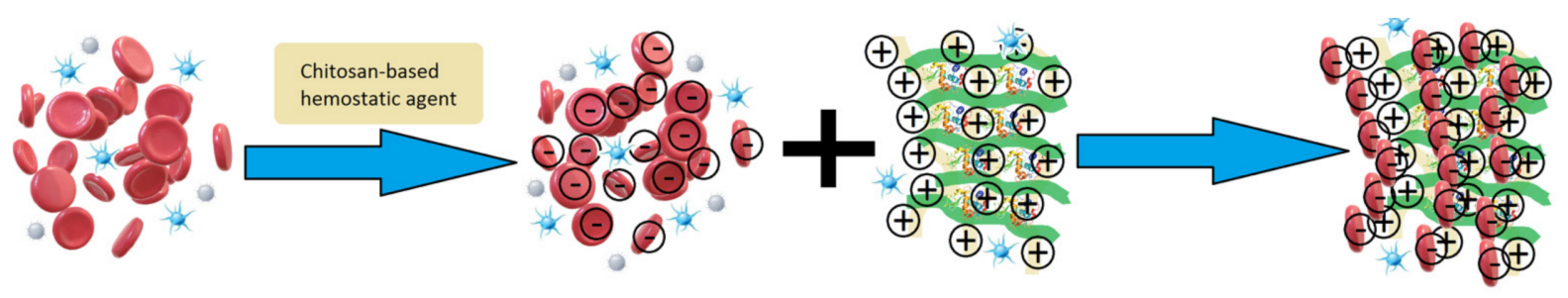

Figure 9. General scheme of hemostatic agent mode of action.

\subsection{Viscosity Study}

The chemical crosslinking resulted in the change of the CS viscosity as given in Figure 10. Native CS has a linear structure and contains $N$-acetylglucosamine as well as glucosamine mers linked with glycosidic bonds. Thus, it easily dissolves in acidic solutions whose viscosity is very low (52 cp). The crosslinking process leads to the formation of cross bindings; thus, a crosslinked structure is obtained. On the other hand, this fact makes the polymer insoluble in aqueous solutions, which instead of dissolving undergoes swelling without losing their shape and structure. To verify the effect of crosslinking conditions 
on the raw CS, newly prepared hydrogels directly after crosslinking were placed in the acetic acid solution, and their viscosity was determined. As one may notice, in the case of samples Gel-1-0 and Gel-2, numerous covalent bonds have been formed, leading to the highly crosslinked and tight structure formation, which resulted in the significantly higher viscosity. Samples Gel-3-0 and Gel-4-0 also were very viscous (800-900 cp). To prepare hemostatic materials, raw hydrogels were lyophilized. The products in their final form were characterized by reduced viscosity, which can be assigned in the change of their porosity and spatial architecture due to the dry-freezing process. It is noteworthy that such viscosity behavior is typical for hydrogel materials and enables distinguishing physical and chemical ones [32-36].

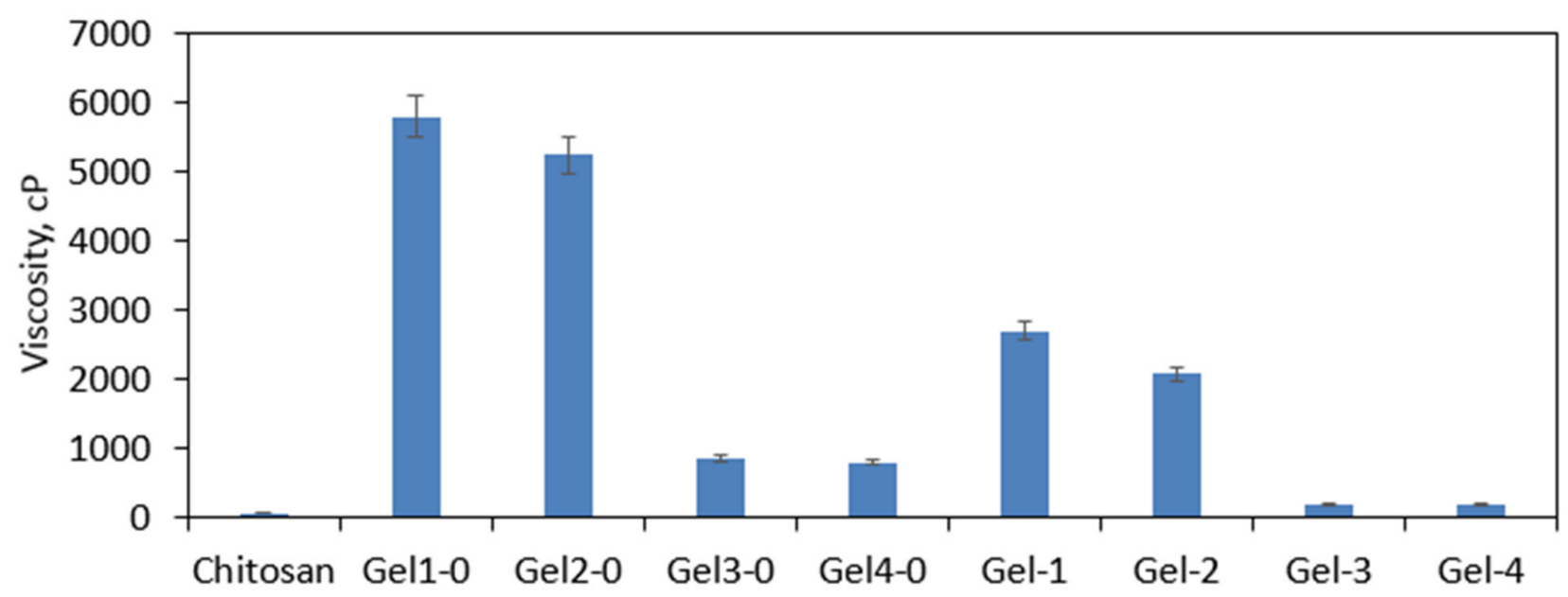

Figure 10. Viscosity study of the raw chitosan, hydrogels after crosslinking (Gel1-0 to Gel4-0), and hydrogels after lyophilization and swelling with distilled water (Gel-1 to Gel-4).

\subsection{Swelling Properties Study}

Hydrogels can be described as hydrophilic materials with a three-dimensional porous structure that can absorb high amounts of water and aqueous solutions. Therefore, their swelling abilities are correlated with the chemical structure as well as porosity and presence of interconnected channels. In this article, using the same raw materials, two different types of potential hemostatic agents have been developed, namely patches (dedicated to broad but shallow wounds) and beads (with the future applications for deep puncture and shotgun wounds). To verify their potential, a study of the swelling properties has been carried out using distilled water, SBF, and human blood (Figure 11). Samples Gel 1 and Gel 2 exhibited superior swelling capacity comparing to other samples, especially in the case of distilled water, which was considered as significantly different. However, it must be acknowledged that absorbing high amounts of the liquid may result in an undesired materials volume increase followed by its structure collapse, which may result as a consequence in the integrity disruption. Samples Gel 3 and 4 are characterized by nearly a twice lower swelling degree in distilled water, whereas this feature in SBF is very similar to the Gel 1 and Gel 2 samples. Such difference can be assigned to the crosslinking agents ratio used for hemostatic agents' preparation. The higher the L-glutamic acid content, the higher the swelling degree that has been obtained, which can be caused by a slightly longer carbon chain providing more space for water molecules after crosslinking. There is a high difference in the sorption abilities comparing various swelling media. The swelling is much lower for SBF and blood, which can be explained by the more complexed chemical composition, especially for the last one, which contains not only various ions but also proteins and other molecules that may interact with amino or hydroxyl groups of the materials due to hydrogen bonds formation, thus hampering water molecules penetration inside the materials' structure [31]. Additionally, there is a high dissimilarity for a patch 
and beads. The hemostatic agents in the form of spheres are characterized by a few times smaller swelling degrees than patches that can be explained by the lower porosity resulted due to differences in fabrication methods. Importantly, in the case of samples swollen with human blood for both forms, the best sorption abilities were obtained for sample no. 2 with the highest glutamic acid content. The sorption properties can be assigned to the presence of multiple hydrophilic moieties present in the modified CS structure as well as incorporated additional groups such as amino and carboxyl ones [31,37]. It is noteworthy that incubation for all samples lasted $5 \mathrm{~min}$, which leads to the conclusion that all samples in such a short period of time are capable of stopping the bleeding and forming an artificial clot [9].

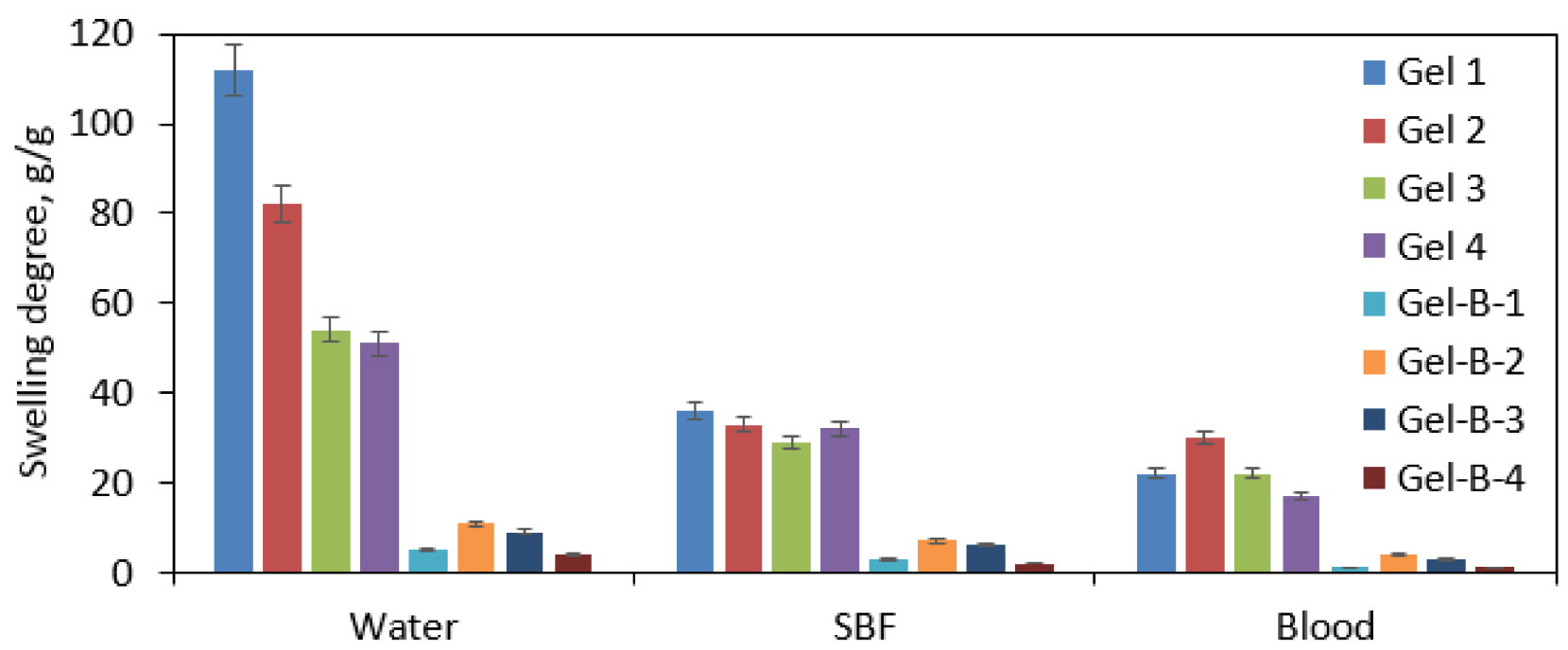

Figure 11. Swelling properties study in different swelling media (distilled water, simulated body fluid (SBF), and blood) of Gel 1-4 hemostatic agents in the form of a patch; Gel-B-1-4 hemostatic agents in the form of beads.

\subsection{Antioxidant Activity Study}

The formation of a wound as a result of skin damage may lead to oxidation stress, which negatively affects the healing process. Therefore, additional biological activity leading to the free radical scavenging is a highly desired feature for modern biomaterials. HemA modified with four different extracts was investigated for its antioxidant activity (Figure 12). The performance of Extracts 1 and 4 as well as samples Gel-1-Extr-4 and Gel-3Extr- 1 can be considered as significantly different. The results given in Figures $3-5$ have shown that all of them contain phenolic and polyphenolic compounds responsible for free radicals' neutralization. To verify this feature, the extracts and two samples characterized by best swelling capacity (Gel 1 and Gel 2) were used to study their antioxidant behavior. The highest biological activity was obtained for commercial extract followed by the extract prepared using $95 \%$ ethanol solution subjected to ultrasounds treatment, which were able to remove almost $50 \%$ of DPPH• (Extract 1 ) and nearly 20\% (Extract 4 ), respectively in only one hour. Further tests were carried out for extracts immobilized inside hemostatic agents matrixes. One may observe that after $60 \mathrm{~min}$, biomaterials have neutralized more than $10 \%$ of free radicals (Extract 1 and 4). For Extracts 2 and 3, the amounts of free radicals being removed were similar for the solutions being tested alone and for ready biomaterials. Kalanchoe pinnata is a plant known for its antioxidant activity, which can be assigned to the phenolic and polyphenolic compounds present in its leaves [24]. On the other hand, CS due to the free amino groups as well as secondary hydroxyl moieties content also possesses the ability of free radicals' removal. The significantly higher antioxidant performance of the raw extract is explained by the easier access to $\mathrm{DPPH} \bullet$, which is much hampered due to the strict and compact form of the hemostatic agents. However, comparing evaluated biomaterials activity to similar products, the antioxidant activity is considerably higher [31]. 


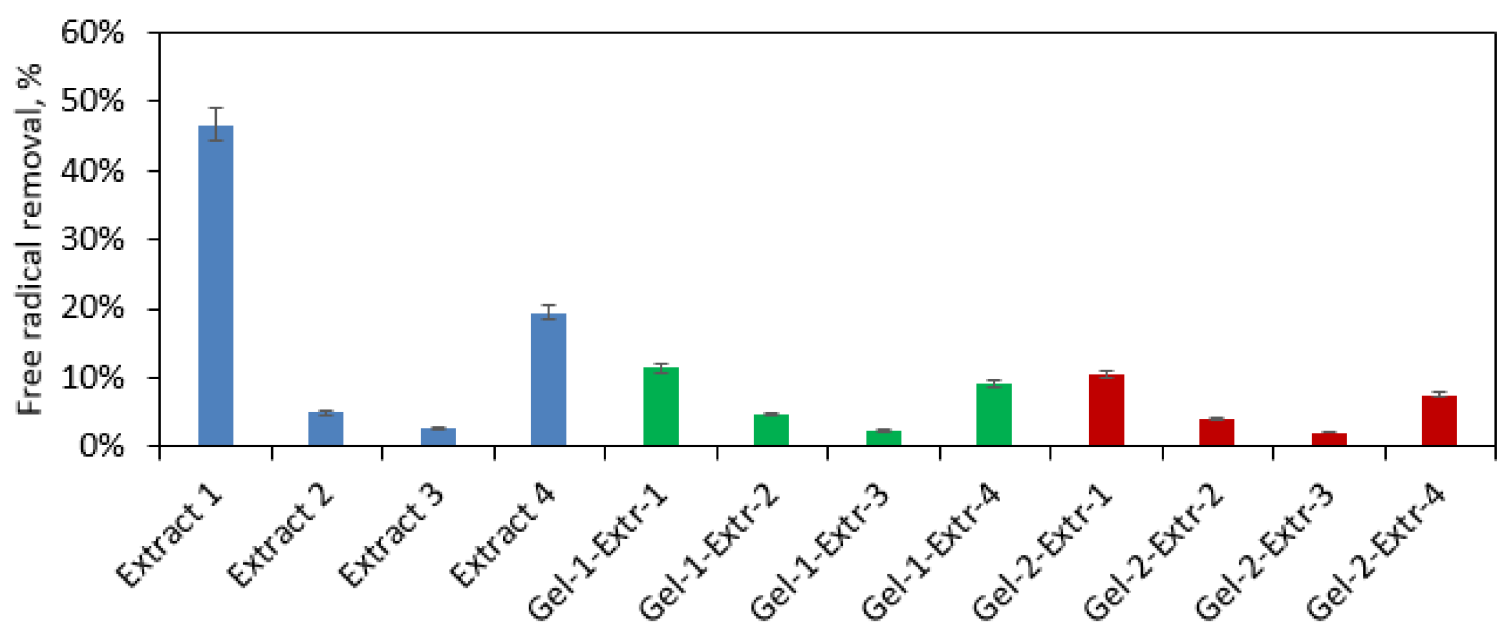

Figure 12. Antioxidant properties study by (2,2-diphenyl-1-picrylhydrazyl) (DPPH) method.

\subsection{Morphology Study}

Prepared gels in the patches form were investigated for their morphology in a dried and swollen form (Figure 13). One may notice that all samples are highly porous and possess numerous interconnected channels that easily fill with the swelling medium. It is noteworthy that due to their superior porosity and a lot of free space inside the 3D matrix after contact with the hydrophilic liquid, they do not increase their volume in a significant manner, since it replaces the air present inside the channels.
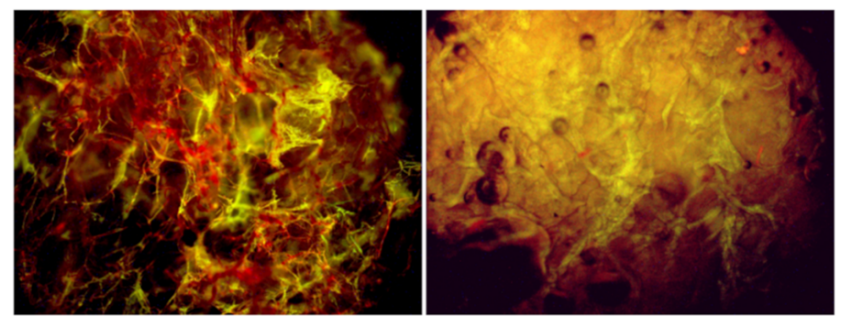

(a)
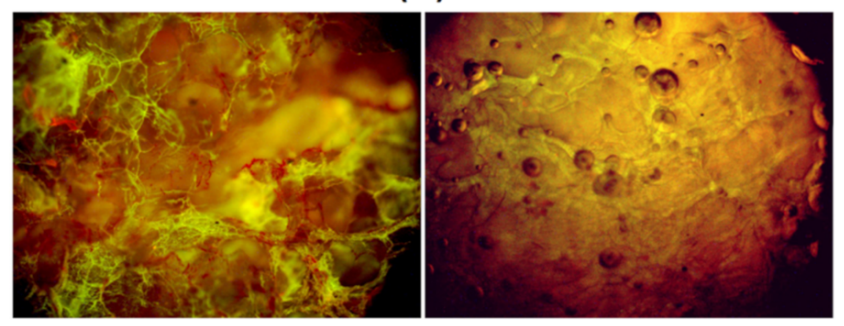

(c)

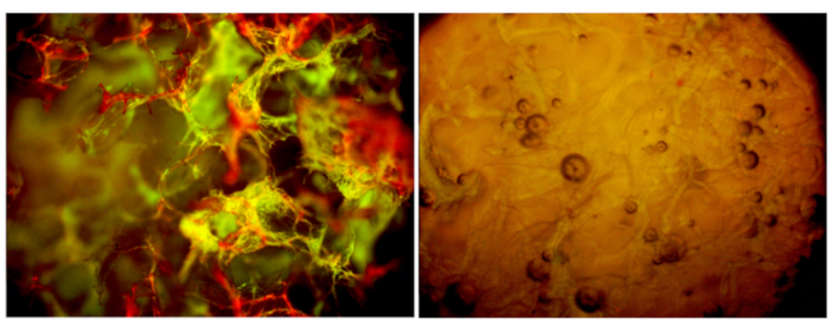

(b)
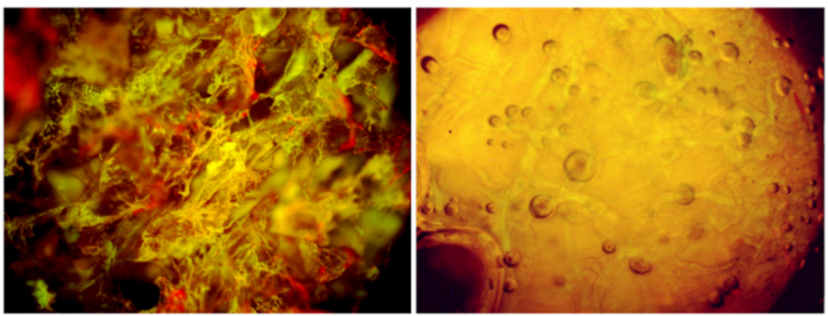

(d)

Figure 13. Microphotographs of the hydrogels (a) Gel-1 dried and swollen; (b) Gel-2 dried and swollen; (c) Gel-3 dried and swollen; (d) Gel-4 dried and swollen.

Figure 14 presents SEM microphotographs of the hemostatic agents in the form of beads. All spheres are characterized by a dense surface. Samples Gel-1-B and Gel-3-B under higher magnification exhibit some roughness, whereas Gel-2-B and Gel-4-B surfaces are smoother but contain "volcano-like" regions, which can be a consequence of the air leaving the bead after a coagulation bath. The lower amount of macropores in a dried form compared to the patch materials explains the lower swelling abilities. 


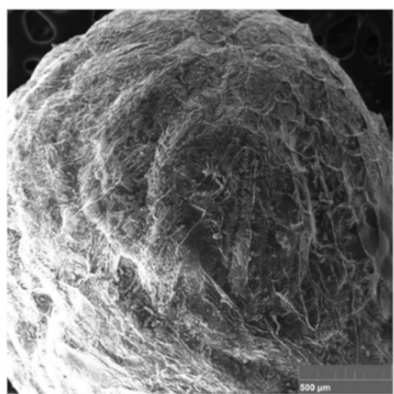

(a)

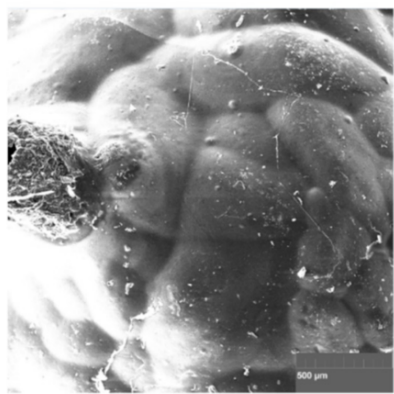

(d)

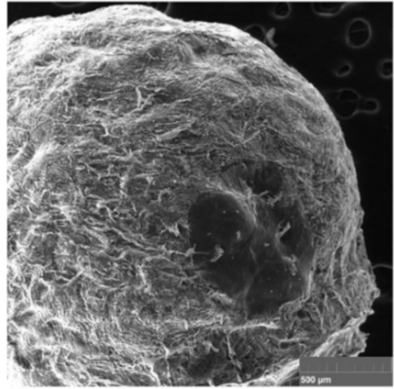

(g)

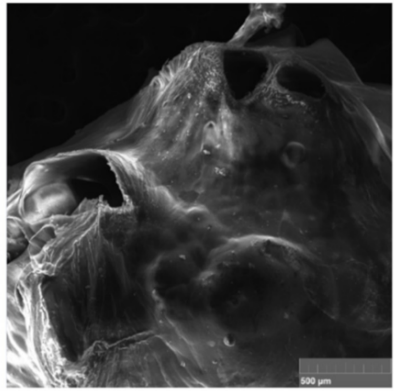

(j)

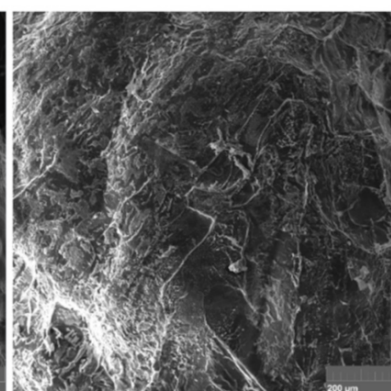

(b)

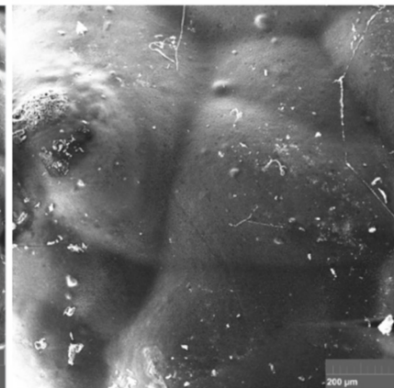

(e)

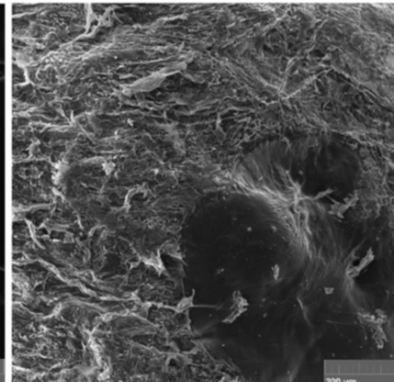

(h)

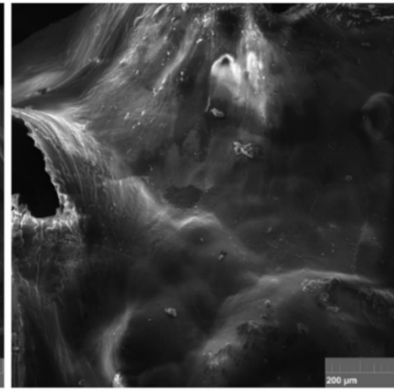

(k)

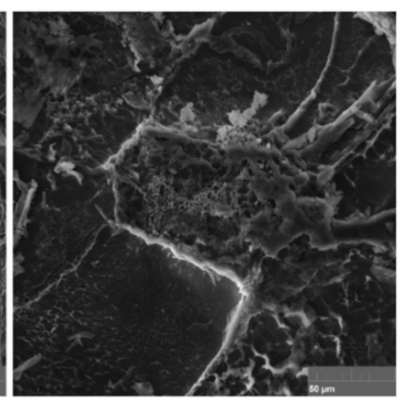

(c)

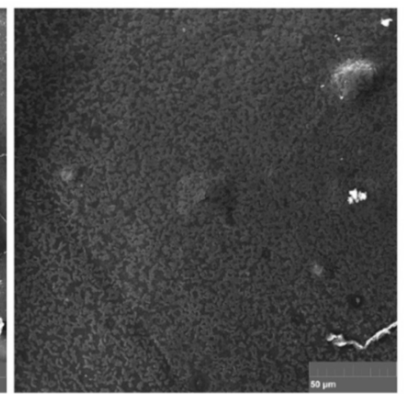

(f)

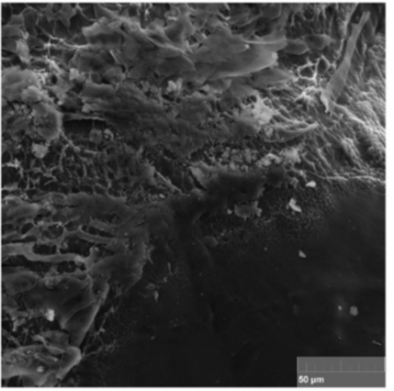

(i)

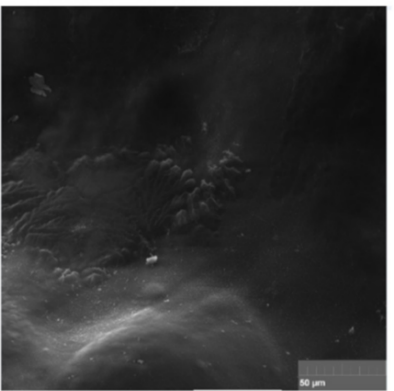

(I)

Figure 14. SEM microphotographs of the chitosan-based beads: (a) Gel-1-B 500×; (b) Gel-1-B 1000× ; (c) Gel-1-B 5000×; (d) Gel-2-B 500×; (e) Gel-2-B 1000×; (f) Gel-2-B 5000×; (g) Gel-3-B 500×; (h) Gel-3-B 1000×; (i) Gel-3-B 5000×; (j) Gel-4-B 500×; (k) Gel-4-B 1000×; (1) Gel-4-B 5000×.

The samples with highest ability of blood sorption were compared before (Figure 15a,b) and after contact with the swelling medium (Figure 15c,d). Both samples Gel-1 and Gel-2 were characterized by a spatial, highly porous structure with high curvature and multiple interconnected channels, which highly contribute to excellent swelling properties, as shown in Figure 11. The pores edges are quite sharp and of petal-like shape. The presence of macropores enables aqueous blood fraction migration inside the three-dimensional structure as well as its stabilization inside the biomaterial. There are no significant differences between Gel-1 and Gel-2, which leads to the conclusion that the chemical composition plays a major role in the swelling capability. Figure $15 \mathrm{c}$,d shows the materials surface after incubation 
with human blood. It is noteworthy that some significant changes may be observed. The surfaces are smoother and covered with protein-like substances. It can be noticed that the surface coverage is higher in the case of Sample Gel-2, which leads to the conclusion that this material interacts stronger with blood components due to electrostatic attractions between positively charged amino groups and negatively charged carboxylic ones. Almost all pores are covered with biomolecules. Such results show that the hemostatic agents 5 min after placement may achieve nearly full sorption capacity, thus potentially blocking the hemorrhage, which is a very important feature for the future applications.

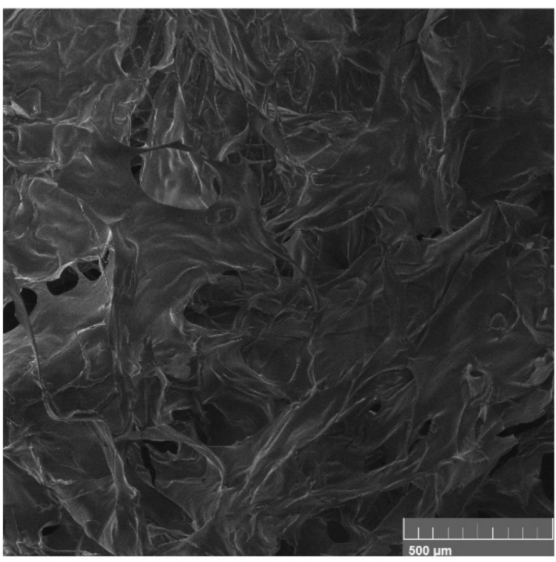

(a)

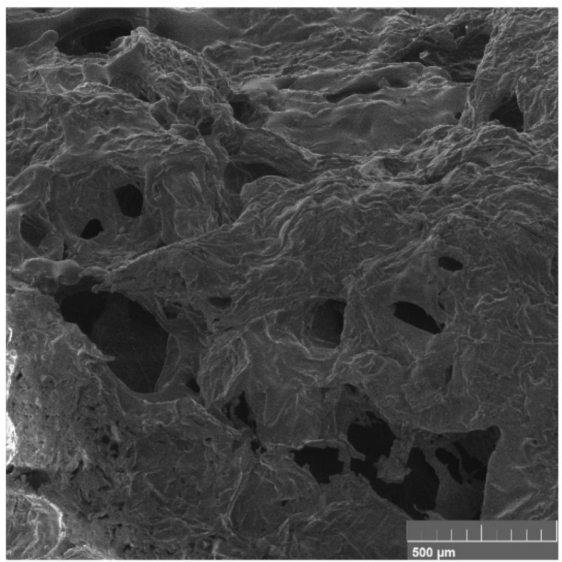

(c)

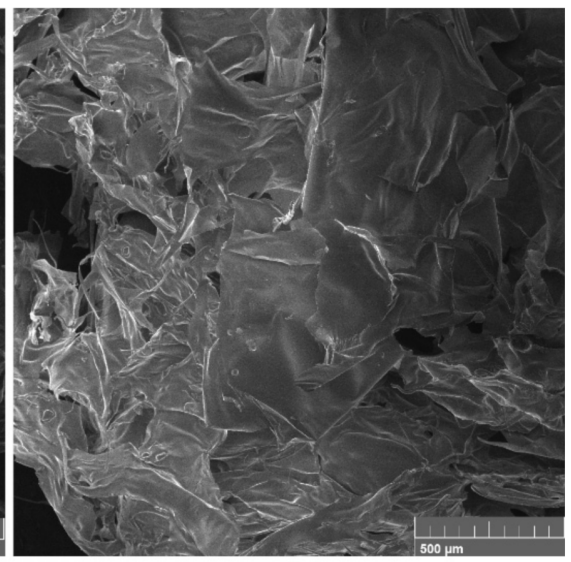

(b)

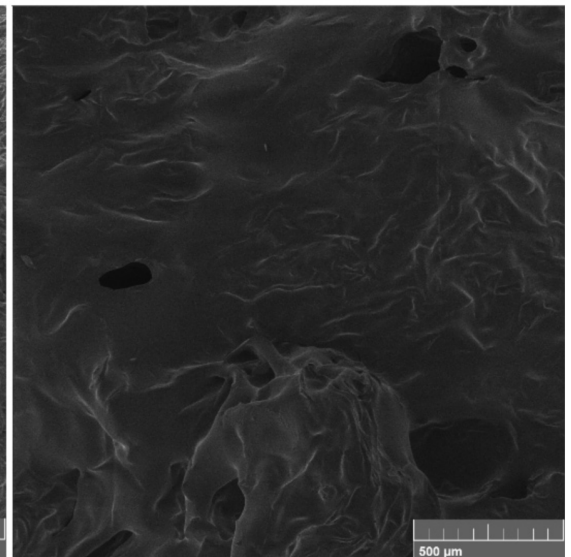

(d)

Figure 15. SEM microphotographs showing surface roughness of (a) Gel-1, (b) Gel-2, and (c) Gel-1 after incubation with blood; (d) Gel-2 after incubation with blood.

Figure 16 shows the samples roughness before $(a, b)$ and after $(c, d)$ incubation with blood. Both samples before the incubation are characterized by very high roughness due to the numerous open-structure pores and their sharp edges. After contact with human fluid due to the material coverage with blood components, the surface is significantly smoother, and the roughness has decreased. It can be observed that the interactions between the swelling medium and material are a little bit different, since it seems that the adhesion is higher in the case of the Gel-2 sample. The sample Gel-1 surface is much smoother after blood contact. The change in the materials surface can be explained by the electrostatic effects leading to the negatively charged molecules attraction and immobilization resulting in the artificial clot formation. Such behaviors are explained by the chemical structure of the CS, which contains free amino groups that interact with erythrocytes and platelets $[9,10,31]$. The incorporation of L-aspartic (C5) and L-glutamic acid resulted in the increase of $\mathrm{NH}_{2}$ moieties which, on the other hand, could lead to the increased electrostatic attractions 
between negatively charged blood components such as proteins and cells, which is crucial during blood clotting cascade and hemostasis restoration [9].

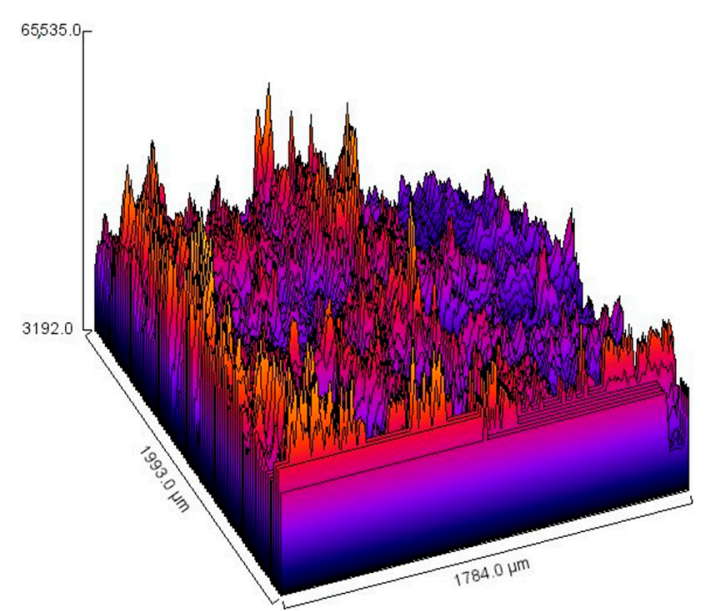

(a)

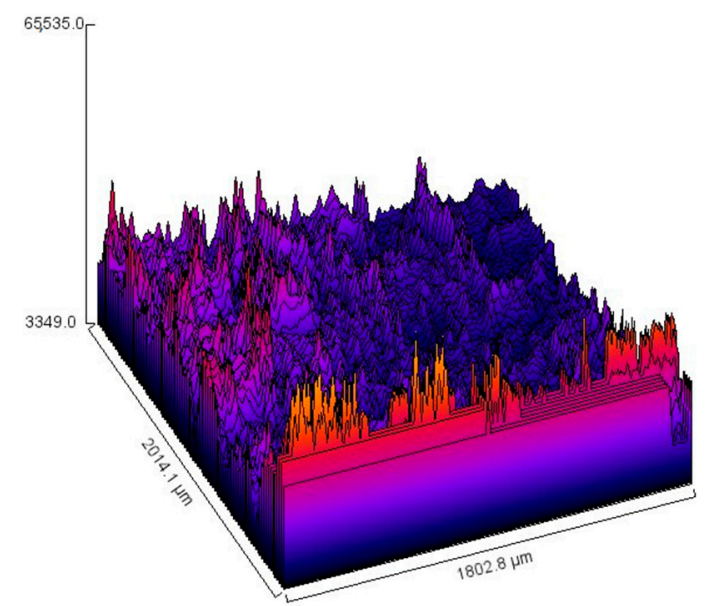

(c)

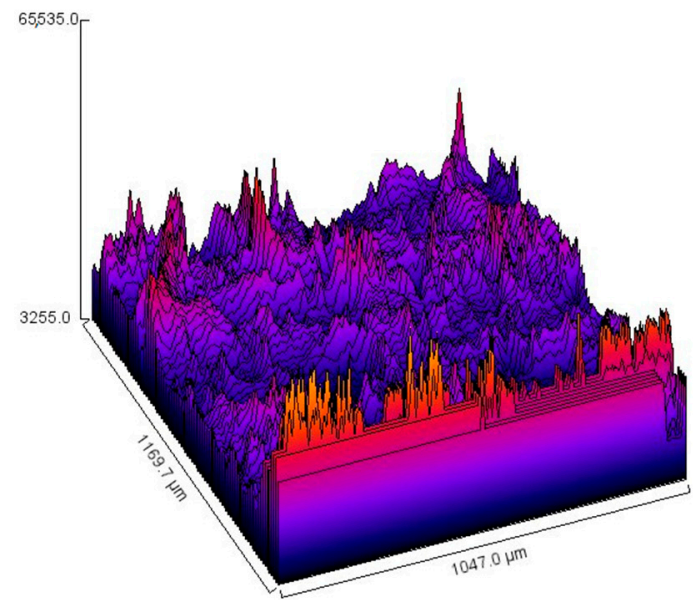

(b)

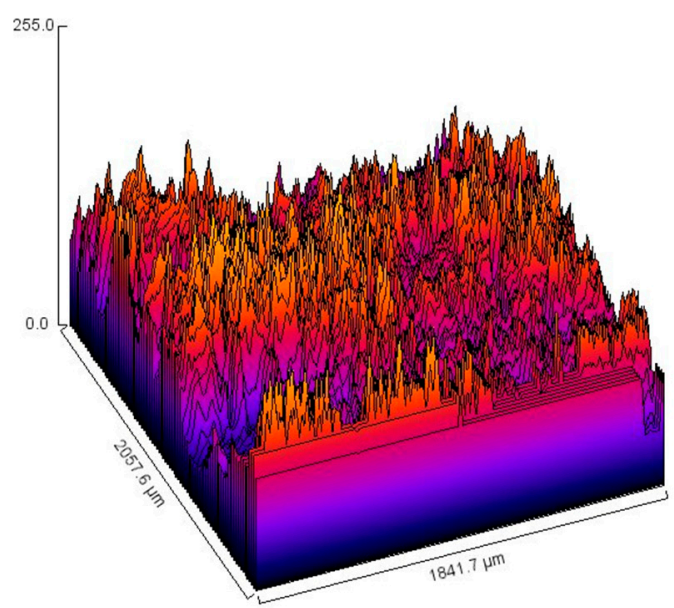

(d)

Figure 16. Surface roughness of the Gel-1 and Gel-2 samples before (a,b) and after incubation with blood (c,d).

\subsection{Mechanical Properties Study}

Biomaterials dedicated for topical applications should be characterized with appropriate mechanical properties to maintain their integrity after swelling with body fluids and not tearing to release smaller fragments, which may contaminate the wound. Hydrogels depending on the polymer used for their preparation are characterized by varied durability. Natural raw materials are mostly characterized by lower resistance to mechanical factors than synthetic ones. Figure 17 shows the results of TS evaluation performed on the "dog bone"-shaped samples swollen with SBF. Raw CS is characterized by low endurance. The crosslinking process leading to the formation of bridges from $L$-aspartic and $L$-glutamic acid molecules between linear chains leading to crosslinked structure formations enhances this feature. A crucial factor affecting the increased durability is the formation of covalent amide bonds between free amino groups coming from CS and carboxylic moieties present in amino acids. Figure 17 shows the tensile strength for the Gel-1, Gel-2, Gel-3, and Gel-4 samples. The highest mechanical durability was obtained for the sample Gel-1, whose TS was above $4 \mathrm{kPa}$ and had a strain of almost $80 \%$. Other samples' TS value were slightly 
below $4 \mathrm{kPa}$. Such results correspond to other researchers' data [38-41] and suggest that the prepared biomaterials durability is at the satisfactory level for topical hemostatic agents' applications.

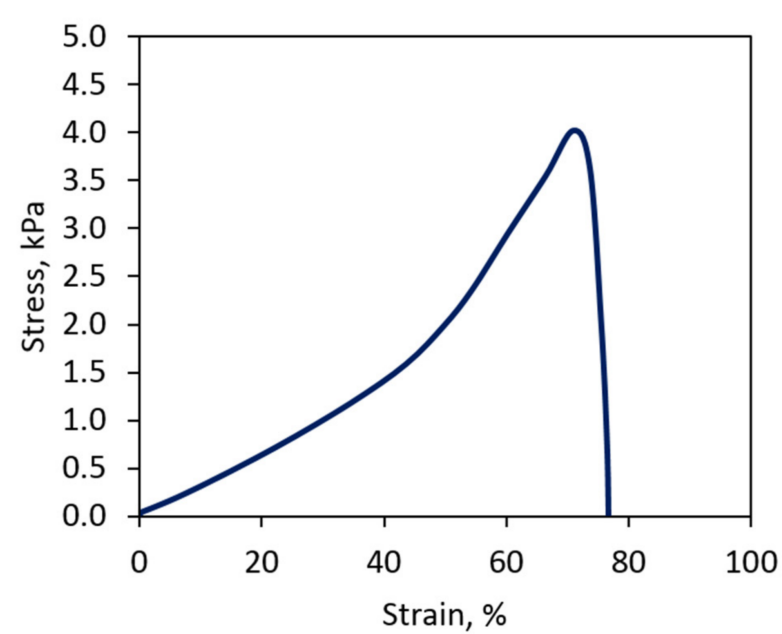

(a)

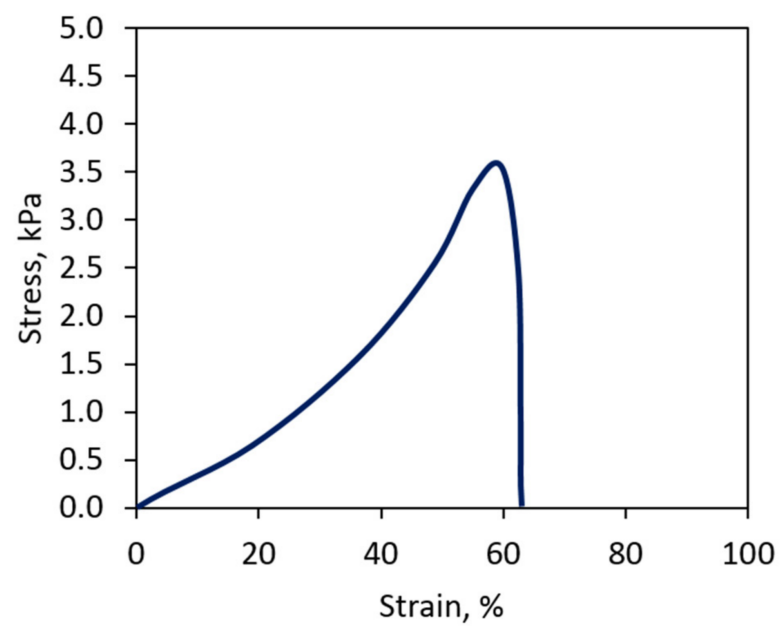

(c)

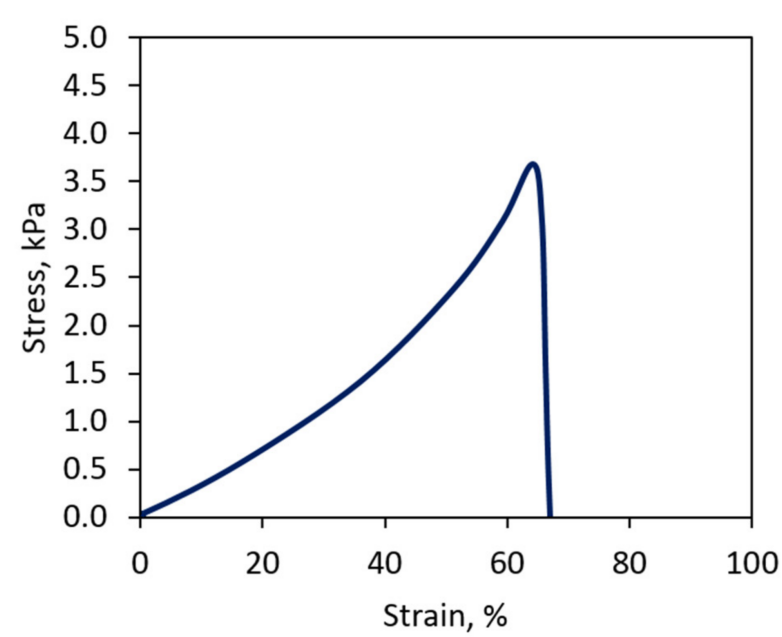

(b)

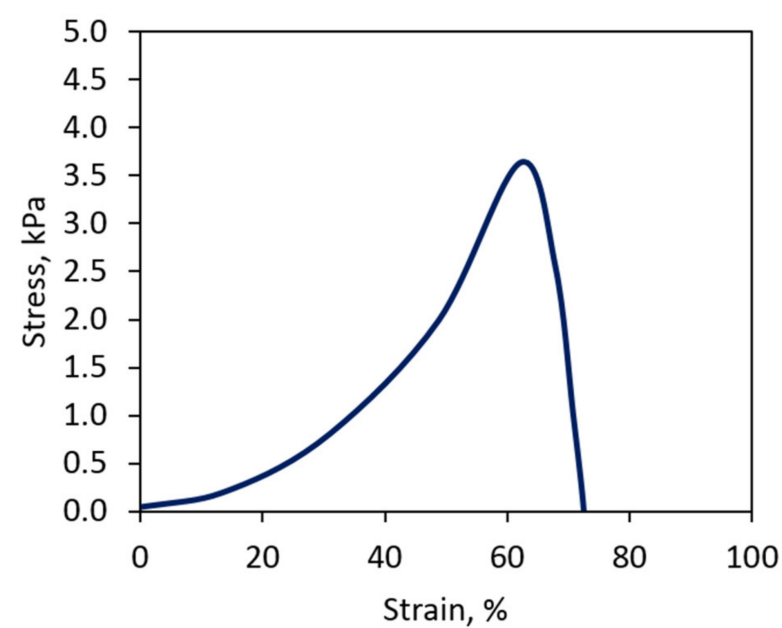

(d)

Figure 17. Mechanical durability of the samples: (a) Gel-1; (b) Gel-2; (c) Gel-3; (d) Gel-4.

\subsection{Cytotoxicity Study}

To investigate in vitro biocompatibility of the prepared materials by the quantitative method, XTT assay was carried out, which is a standard procedure for medical devices. According to the ISO 10993 norm, the biomaterial is non-cytotoxic when the number of viable cells is not lower than $70 \%$ compared to the control. CS is well-known for its biocompatibility, which can be assigned to the structural similarities to glycosaminoglycans such as chondroitin sulfate, which is one of the natural ECM components. It can be observed that the presence of pristine CS has a positive impact on cells' proliferation (Figure 18). It is noteworthy that the chemical modification of this biopolymer structure may deteriorate this property, which can be e.g., a result of toxic crosslinking agents' release to the cell culture medium. Figure 18 shows that all of the prepared samples do not cause any negative effect on the number of viable cells. On the contrary, in the case of samples Gel-1 and Gel-2, the number of alive cells is higher by almost $50 \%$. The viable percentage of the Gel-3 and Gel-4 samples was slightly lower yet still higher than that of native CS [31,42]. 


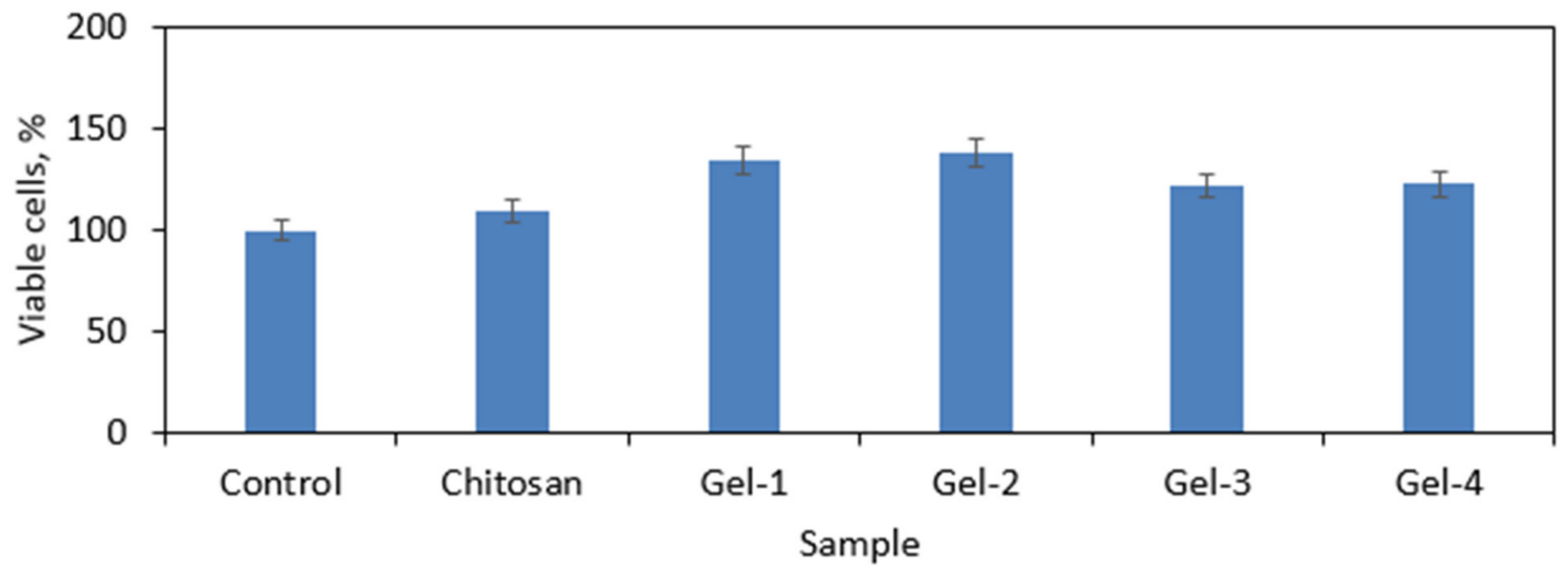

Figure 18. Cytotoxicity study performed using (2,3-Bis-(2-Methoxy-4-Nitro-5-Sulfophenyl)-2H-Tetrazolium-5Carboxanilide) (XTT) assay.

The lack of cytotoxicity was further studied by cells' morphology investigations (qualitative method) after $72 \mathrm{~h}$ of cell culture. As shown in Figure 19, after 3 days, the cells formed monolayers. The fibroblasts were flattened and adhered to the flask bottom. The cells were characterized by a spindle-like shape typical for L929. There are a few rounded cells present. No detached fibroblasts are visible. Therefore, the biomaterials can be described as non-cytotoxic to the skin cells [31,42].

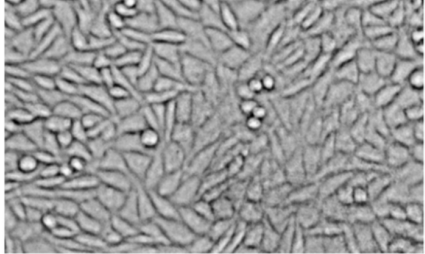

(a)

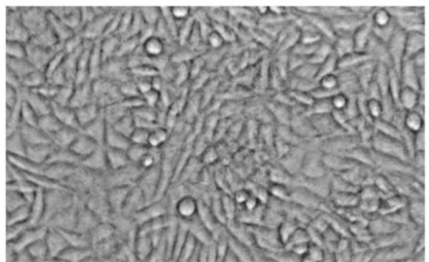

(d)

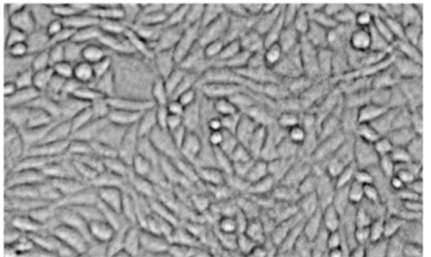

(b)

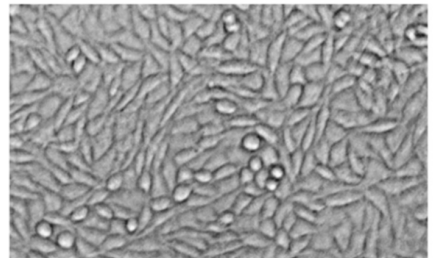

(e)

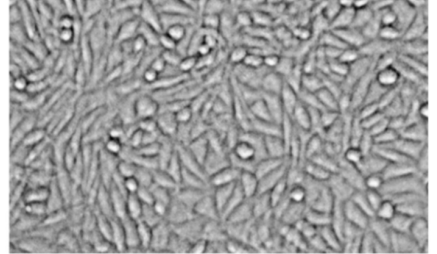

(c)

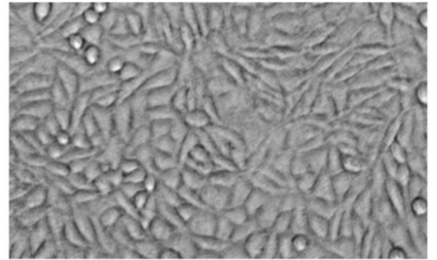

(f)

Figure 19. Fibroblasts' morphology study: (a) control; (b) raw chitosan; (c) Gel-1; (d) Gel-2; (e) Gel-3; (f) Gel-4.

\subsection{Future Perspectives}

Performed studies showed that the application of fungal CS and other natural components led to the preparation of novel biomaterials with interesting properties. The choice of non-animal raw materials increases their potential use to patients suffering from allergy to shellfish. Importantly, the products can be obtained from cheap and locally available resources. Due to the satisfying biological features of the potential hemostatic agents, further actions will be focused on in vivo tests on small animal models. The positive effect on skin cells suggests that future application should mostly manage superficial hemorrhages, since the biomaterial may contribute to faster healing. On the other hand, obtainment of the products in the spherical shape gives the possibility of their use in deep gunshot or stab wounds. Before their commercial application, the biomaterials should undergo upscaling, which can be a challenging process due to the risk of local overheating spots appearing during the crosslinking procedure, which is a common problem associated with 
microwave-assisted synthesis. This phenomenon may lead to some problems with the repeatability of the samples and their homogeneity. Another issue related to microwaves is their penetration depth, which must be taken under consideration when choosing an appropriate reactor type. One possible limitation is also the risk of insufficient accessibility of the raw materials, which would meet all of the requirements for medical devices, since currently commercially available CS-based biomaterials are produced mostly using shellfish as a substrate, not fungi. However, the choice of an appropriate reaction vessel as well as stirring method probably will help to overcome this issue.

\section{Conclusions}

The aim of the following research was to obtain novel naturally derived blood clotting agents in the form of dressing and beads using fungal chitosan as a starting material, which was subjected to successful crosslinking with amino acids under microwave-assisted conditions followed by modification with four different Kalanchoe pinnata extracts to increase the antioxidant activity of the potential hemostatic biomaterials. Their shape was successfully processed to both patch and beads forms. Moreover, the products retained favorable features of native chitosan. The resulted products were investigated for their chemical structure and composition, confirming increased free amino groups content and phenolic/polyphenolic compounds presence. A morphology study showed spatial and porous architecture responsible for sorption capacity. The biomaterials in form of the patch exhibited superior swelling properties after incubation with human blood, whose components have been proven to interact with the evaluated samples surface. They exhibited satisfactory mechanical durability. Their ability of free radicals scavenging was proved by the DPPH method. Their lack of cytotoxicity confirmed by XTT assay and morphology study shows their potential in the future applications as hemostatic agents.

Author Contributions: Conceptualization, J.R.-P. and Ł.J.; methodology, J.R.-P. and Ł.J.; investigation, J.R.-P., Ł.J., A.S., E.S., and T.G.; resources, M.T., Ł.J., M.P., and J.R.-P.; writing-original draft and preparation, J.R.-P.; writing and reviewing, J.R.-P., Ł.J. and M.P.; visualization, E.S.; supervision, M.P.; M.T. and D.B. project administration, J.R.-P., funding acquisition, J.R.-P. All authors have read and agreed to the published version of the manuscript.

Funding: This research was funded by National Centre for Research and Development, grant number LIDER/21/0120/L-10/18/NCBR/2019 and the APC was funded by National Centre for Research and Development, grant number LIDER/21/0120/L-10/18/NCBR/2019.

Institutional Review Board Statement: Not applicable.

Informed Consent Statement: Not applicable.

Data Availability Statement: The data presented in this study are available on request from the corresponding author.

Acknowledgments: Not applicable.

Conflicts of Interest: The authors declare no conflict of interest.

\section{References}

1. Allotey, J.K.; King, A.H.; Kumins, N.H.; Wong, V.L.; Harth, K.C.; Cho, J.S.; Kashyap, V.S. Systematic Review of Hemostatic Agents used in Vascular Surgery. J. Vasc. Surg. 2020, in press. [CrossRef]

2. Bracey, A.; Shander, A.; Aronson, S.; Boucher, B.A.; Calcaterra, D.; Chu, M.W.A.; Culbertson, R.; Jabr, K.; Kehlet, H.; Lattouf, O.; et al. The Use of Topical Hemostatic Agents in Cardiothoracic Surgery. Ann. Thorac. Surg. 2017, 104, 353-360. [CrossRef]

3. Galanakis, I.; Vasdev, N.; Soomro, N.N. A Review of Current Hemostatic Agents and Tissue Sealants Used in Laparoscopic Partial Nephrectomy. Rev. Urol. 2011, 13, 131-138. [PubMed]

4. Nakielski, P.; Pierini, F. Blood interactions with nano- and microfibers: Recent advances, challenges and applications in nano- and microfibrous hemostatic agents. Acta Biomater. 2019, 84, 63-76. [CrossRef] [PubMed]

5. Wang, Y.; Zhao, Y.; Qiao, L.; Zou, F.; Xie, Y.; Zheng, Y.; Chao, Y.; Yang, Y.; He, W.; Yang, S. Cellulose fibers-reinforced self-expanding porous composite with multiple hemostatic efficacy and shape adaptability for uncontrollable massive hemorrhage treatment. Bioact. Mater. 2021, 6, 2089-2104. [CrossRef] [PubMed] 
6. Guajardo, S.; Figueroa, T.; Borges, J.; Aguayo, C.; Fernández, K. Graphene oxide-gelatin aerogels as wound dressings with improved hemostatic properties. Mater. Today Chem. 2021, 20, 100418. [CrossRef]

7. Lv, C.; Li, L.; Jiao, Z.; Yan, H.; Wang, Z.; Wu, Z.; Guo, M.; Wang, Y.; Zhang, P. Improved hemostatic effects by Fe3+ modified biomimetic PLLA cotton-like mat via sodium alginate grafted with dopamine. Bioact. Mater. 2021, 6, 2346-2359. [CrossRef]

8. Gan, C.; Hu, H.; Meng, Z.; Zhu, X.; Gu, R.; Wu, Z.; Wang, H.; Wang, D.; Gan, H.; Wang, J.; et al. Characterization and Hemostatic Potential of Two Kaolins from Southern China. Molecules 2019, 24, 3160. [CrossRef]

9. Pogorielov, V.M.; Sikora, V.Z. Chitosan as a Hemostatic Agent: Current State. Eur. J. Med. Ser. B 2015, 2, 24-33. [CrossRef]

10. Whang, H.S.; Kirsch, W.; Zhu, Y.H.; Yang, C.Z.; Hudson, S.M. Hemostatic Agents Derived from Chitin and Chitosan. J. Macromol. Sci. Polym. Rev. 2005, 45, 309-323. [CrossRef]

11. Lestari, W.; Yusry, W.N.A.W.; Haris, M.S.; Jaswir, I.; Idrus, E. A glimpse on the function of chitosan as a dental hemostatic agent. Jpn. Dent. Sci. Rev. 2020, 56, 147-154. [CrossRef]

12. Kadam, A.A.; Shinde, S.K.; Ghodake, G.S.; Saratale, G.D.; Saratale, R.G.; Sharma, B.; Hyun, S.; Sung, J.-S. Chitosan-Grafted Halloysite Nanotubes- $\mathrm{Fe}_{3} \mathrm{O}_{4}$ Composite for Laccase-Immobilization and Sulfamethoxazole-Degradation. Polymers 2020, $12,2221$. [CrossRef]

13. Koumentakou, I.; Terzopoulou, Z.; Michopoulou, A.; Kalafatakis, I.; Theodorakis, K.; Tzetzis, D.; Bikiaris, D. Chitosan dressings containing inorganic additives and levofloxacin as potential wound care products with enhanced hemostatic properties. Int. J. Biol. Macromol. 2020, 162, 693-703. [CrossRef]

14. Lovskaya, D.; Menshutina, N.; Mochalova, M.; Nosov, A.; Grebenyuk, A. Chitosan-Based Aerogel Particles as Highly Effective Local Hemostatic Agents. Production Process and In Vivo Evaluations. Polymers 2020, 12, 2055. [CrossRef]

15. Li, T.-T.; Lou, C.-W.; Chen, A.-P.; Lee, M.-C.; Ho, T.-F.; Chen, Y.-S.; Lin, J.-H. Highly Absorbent Antibacterial Hemostatic Dressing for Healing Severe Hemorrhagic Wounds. Materials 2016, 9, 793. [CrossRef]

16. Zhong, Q.-K.; Wu, Z.-Y.; Qin, Y.-Q.; Hu, Z.; Li, S.-D.; Yang, Z.-M.; Li, P.-W. Preparation and Properties of Carboxymethyl Chitosan/Alginate/Tranexamic Acid Composite Films. Membranes 2019, 9, 11. [CrossRef]

17. Hu, Z.; Lu, S.; Cheng, Y.; Kong, S.; Li, S.; Li, C.; Yang, L. Investigation of the Effects of Molecular Parameters on the Hemostatic Properties of Chitosan. Molecules 2018, 23, 3147. [CrossRef]

18. Wang, Y.; Yin, M.; Zheng, X.; Li, W.; Ren, X. Chitosan/mesoporous silica hybrid aerogel with bactericidal properties as hemostatic material. Eur. Polym. J. 2021, 142, 110132. [CrossRef]

19. Majaz, Q.A.; Tatiya, A.U.; Khurshid, M.; Nazim, S.; Siraj, S. The miracle plant (Kalanchoe pinnata): A phytochemical and pharmacological review. Int. J. Res. Ayurveda Pharm. 2016, 14, 1-10.

20. Faboro, E.O.; Wei, L.; Liang, S.; McDonald, A.G.; Obafemi, C.A. Hepatoprotective activity of leaves of Kalanchoe pinnata Pers. J. Ethnopharmacol. 2003, 86, 197-202.

21. Dantas de Araújo, E.R.; Félix-Silva, J.; Xavier-Santos, J.B.; Morais Fernandes, J.; Guerra, G.C.B.; de Araújo, A.A.; de Souza Araújo, D.F.; de Santis Ferreira, L.; Júnior, A.S.; Fernandes-Pedrosa, M.-F.; et al. Local anti-inflammatory activity: Topical formulation containing Kalanchoe brasiliensis and Kalanchoe pinnata leaf aqueous extract. Biomed. Pharmacother. 2019, 113, 108721. [CrossRef] [PubMed]

22. Agarwal, H.; Shanmugam, V.K. Anti-inflammatory activity screening of Kalanchoe pinnata methanol extract and its validation using a computational simulation approach. Inform. Med. Unlocked. 2019, 14, 6-14. [CrossRef]

23. Mora-Pérez, A.; Hernández-Medel, M.D.R. Anticonvulsant activity of methanolic extract from Kalanchoe pinnata (Lam.) stems and roots in mice: A comparison to diazepam. Neurologia 2016, 31, 161-168. [CrossRef] [PubMed]

24. Menon, N.; Sparks, J.; Omoruyi, F. Hypoglycemic and hypocholesterolemic activities of the aqueous preparation of Kalanchoe pinnata leaves in streptozotocin-induced diabetic rats. Asian Pac. J. Trop. Biomed. 2015, 5, 3-9. [CrossRef]

25. De Araújo, E.R.D.; Guerra, G.C.B.; Araújo, D.F.d.S.; De Araújo, A.A.; Fernandes, J.M.; De Araújo Júnior, R.F.; Da Silva, V.C.; De Carvalho, T.G.; Ferreira, L.D.S.; Zucolotto, S.M. Gastroprotective and Antioxidant Activity of Kalanchoe brasiliensis and Kalanchoe pinnata Leaf Juices against Indomethacin and Ethanol-Induced Gastric Lesions in Rats. Int. J. Mol. Sci. 2018, $19,1265$. [CrossRef]

26. Faboro, E.O.; Wei, L.; Liang, S.; McDonald, A.G.; Obafemi, C.A. Phytochemical Analyzes from the Leaves of Bryophyllum pinnatum. EJMP 2006, 14, 1-10. [CrossRef]

27. Gabriel, H.; Agbor, A.; Vinson, J.A.; Donnelly, P.E. Folin-Ciocalteau Reagent for Polyphenolic Assay. Int. J. Food Sci. Nutr. Diet. 2014, 3, 147-156.

28. Bezuneh, T.T.; Kebede, E.M. UV—Visible Spectrophotometric Quantification of Total Polyphenol in Selected Fruits. Int. J. Food Sci. Nutr. 2015, 4, 397-401. [CrossRef]

29. Abbas, O.; Compère, G.; Larondelle, Y.; Pompeu, D.; Rogez, H.; Baeten, V. Phenolic compound explorer: A mid-infrared spectroscopy database. Vib. Spectrosc. 2017, 92, 111-118. [CrossRef]

30. Hama, T.; Kouchi, A.; Watanabe, N.; Enami, S.; Shimoaka, T.; Hasegawa, T. In Situ Nondestructive Analysis of Kalanchoe pinnata Leaf Surface Structure by Polarization-Modulation Infrared Reflection-Absorption Spectroscopy. J. Phys. Chem. B 2017, 121, 11124-11131. [CrossRef]

31. Radwan-Pragłowska, J.; Piątkowski, M.; Deineka, V.; Janus, Ł.; Korniienko, V.; Husak, E.; Holubnycha, V.; Liubchak, I.; Zhurba, V.; Sierakowska, A.; et al. Chitosan-Based Bioactive Hemostatic Agents with Antibacterial Properties-Synthesis and Characterization. Molecules 2019, 24, 2629. [CrossRef] 
32. Bartnikowski, M.; Wellard, M.; Woodruff, M.A.; Klein, T.J. Tailoring Hydrogel Viscoelasticity with Physical and Chemical Crosslinking. Polymers 2015, 7, 2650-2669. [CrossRef]

33. Park, J.K.; Nah, J.-W.; Choi, C. Thermosensitive Chitosan-based Hydrogel with Growth Factor as Adhesion Barrier. Polym. Korea 2015, 39, 480-486. [CrossRef]

34. Franzén, H.M.; Draget, K.I.; Langebäck, J.; Nilsen-Nygaard, J. Characterization and Properties of Hydrogels Made from Neutral Soluble Chitosans. Polymers 2015, 7, 373-389. [CrossRef]

35. Bashir, S.; Teo, Y.Y.; Ramesh, S.; Ramesh, K.; Rizwan, M.; Rizwan, M. Synthesis and characterization of pH-sensitive N-succinyl chitosan hydrogel and properties for biomedical applications. J. Chil. Chem. Soc. 2019, 64, 0717-9707. [CrossRef]

36. Costa, C.N.; Teixeira, V.G.; Delpech, M.C.; Souza, J.V.; Costa, M.A. Viscometric study of chitosan solutions in acetic acid/sodium acetate and acetic acid/sodium chloride. Carbohydr. Polym. 2015, 133, 245-250. [CrossRef]

37. Rohindra, T.R.; Nand, A.V.; Khurma, J.R. Swelling properties of chitosan hydrogels. SPJNAS 2004, 22, 32-35. [CrossRef]

38. Wahba, M.I. Enhancement of the mechanical properties of chitosan. J. Biomater. Sci. Polym. Ed. 2019, 31, 350-375. [CrossRef]

39. Le, H.R.; Qu, S.; Mackay, R.E.; Rothwell, R. Fabrication and mechanical properties of chitosan composite membrane containing hydroxyapatite particles. J. Adv. Ceram. 2012, 1, 66-71. [CrossRef]

40. Grzabka-Zasadzińska, A.; Amietszajew, T.; Borysiak, S. Thermal and mechanical properties of chitosan nanocomposites with cellulose modified in ionic liquids. J. Therm. Anal. Calorim. 2017, 130, 143-154. [CrossRef]

41. Notin, L.; Viton, C.; David, L.; Alcouffe, P.; Rochas, C.; Domard, A. Morphology and mechanical properties of chitosan fibers obtained by gel-spinning: Influence of the dry-jet-stretching step and ageing. Acta Biomater. 2006, 2, 387-402. [CrossRef]

42. Huang, M.; Khor, E.; Lim, L.-Y. Uptake and cytotoxicity of chitosan molecules and nanoparticles: Effects of molecular weight and degree of deacetylation. Pharm. Res. 2004, 21, 344-353. [CrossRef] 